\title{
Autophagy orchestrates the regulatory program of tumor-associated myeloid-derived suppressor cells
}

\author{
Themis Alissafi, ${ }^{1}$ Aikaterini Hatzioannou, ${ }^{1}$ Konstantinos Mintzas, ${ }^{1}$ Roza Maria Barouni, ${ }^{1}$ Aggelos Banos, ${ }^{1}$ Sundary Sormendi, ${ }^{2}$ \\ Alexandros Polyzos, ${ }^{1}$ Maria Xilouri, ${ }^{1}$ Ben Wielockx, ${ }^{2}$ Helen Gogas, ${ }^{3}$ and Panayotis Verginis ${ }^{1}$ \\ 'Biomedical Research Foundation of the Academy of Athens, Athens, Greece. 'Department of Clinical Pathobiochemistry, Institute for Clinical Chemistry and Laboratory Medicine and Department \\ of Internal Medicine, University Dresden, Dresden, Germany. ${ }^{3}$ First Department of Medicine, National and Kapodistrian University of Athens, School of Medicine, Athens, Greece.
}

\begin{abstract}
Myeloid-derived suppressor cells (MDSCs) densely accumulate into tumors and potently suppress antitumor immune responses, promoting tumor development. Targeting MDSCs in tumor immunotherapy has been hampered by lack of understanding of the molecular pathways that govern MDSC differentiation and function. Herein, we identify autophagy as a crucial pathway for MDSC-mediated suppression of antitumor immunity. Specifically, MDSCs in patients with melanoma and mouse melanoma exhibited increased levels of functional autophagy. Ablation of autophagy in myeloid cells markedly delayed tumor growth and endowed antitumor immune responses. Notably, tumor-infiltrating autophagy-deficient monocytic MDSCs (M-MDSCs) demonstrated impaired suppressive activity in vitro and in vivo, whereas transcriptome analysis revealed substantial differences in genes related to lysosomal function. Accordingly, autophagy-deficient M-MDSCs exhibited impaired lysosomal degradation, thereby enhancing surface expression of MHC class II molecules, resulting in efficient activation of tumor-specific CD4+ $\mathrm{T}$ cells. Finally, targeting of the membrane-associated RING-CH1 (MARCH1) E3 ubiquitin ligase that mediates the lysosomal degradation of MHC II in M-MDSCs attenuated their suppressive function, and resulted in markedly decreased tumor volume followed by development of a robust antitumor immunity. Collectively, these findings depict autophagy as a molecular target of MDSC-mediated suppression of antitumor immunity.
\end{abstract}

\section{Introduction}

The effectiveness of current cancer immunotherapies is critically dependent on the presence of activated effector $\mathrm{T}$ cells in the tumor microenvironment (TME), and preexisting $\mathrm{T}$ cell infiltration in solid tumors holds a prognostic value because it is associated with clinical response (1). Despite the operation of immune surveillance mechanisms, tumors form endowed immunosuppressive networks that impede the elicitation of potent antitumor immune responses and impair the success of immunotherapy (2). Myeloidderived suppressor cells (MDSCs), the progenitors of dendritic cells, macrophages, and neutrophils, comprise a major component of the tumor-induced immunosuppressive circuit. In mice, MDSCs are characterized as $\mathrm{Gr}-\mathrm{1}^{+} \mathrm{CD} 11 \mathrm{~b}^{+}$cells and can be further divided based on their morphology as monocytic (M-MDSCs, CD11b+Ly6G Ly6 $\mathrm{C}^{\mathrm{hi}}$ ) or granulocytic (G-MDSCs, CD11b $\left.{ }^{+} \mathrm{Ly} 6 \mathrm{G}^{+} \mathrm{Ly} 6 \mathrm{C}^{\mathrm{lo}}\right)$, whereas in humans, MDSCs are frequently characterized as HLA-DR ${ }^{\mathrm{lo}-}$ $\mathrm{CD}^{-}{ }^{-} \mathrm{CD} 33^{+} \mathrm{CD}^{+} 5^{+}$cells (3-5). Multiple mechanisms have been attributed to MDSC-mediated inhibition of antitumor immune responses, ranging from secretion of immunosuppressive mediators to direct cell-to-cell contact (6-7). To date, major therapeutic efforts in cancer aim to switch the differentiation and function of MDSCs toward an immunogenic phenotype.

Authorship note: TA and AH contributed equally to this work. Conflict of interest: The authors have declared that no conflict of interest exists. Submitted: March 5, 2018; Accepted: June 14, 2018.

Reference information: / Clin Invest. 2018;128(9):3840-3852. https://doi.org/10.1172/JCI120888.
Autophagy is a fundamental lysosomal catabolic pathway involving degradation of unwanted proteins and organelles to maintain nutrient and cell homeostasis (8). The autophagy pathway is induced under hypoxic conditions, and hypoxia is a cardinal feature of most tumors that possesses a major role in tumor progression, metastasis, and response to therapy (9). Although most studies have focused on the role of autophagy in tumor cells (10), how this pathway affects the immune components of the TME and specifically MDSCs remains unknown. Several lines of evidence, however, have indirectly shown autophagy to be a major regulator of MDSC function. To this end, hypoxia-inducible factor $1 \alpha$ (HIF-1 $\alpha$ ), which regulates autophagy, has been demonstrated to promote MDSC differentiation toward tumor-associated macrophages (11-13). Moreover, high-mobility group box protein 1 has been shown to promote MDSC survival through induction of autophagy and pharmacologic inhibition of autophagy-induced MDSC cell death (14). In a similar fashion, endoplasmic reticulum (ER) stress that activates autophagy as a compensatory mechanism for cell survival has been linked to TRAIL-R-mediated MDSC apoptosis (15-17). Finally, ROS are central in inducing autophagy and have been demonstrated to regulate the stress sensor CHOP in MDSCs that facilitates MDSC accumulation and function (18, 19), suggesting that autophagy might be implicated in MDSC suppression. Although all aforementioned reports indicate a possible link of autophagy to the function of tumor-associated MDSCs, a direct role of this pathway as well as the precise autophagy-related molecular events in MDSC-mediated suppression of antitumor immunity remain elusive. 
In this study, we delineate an important role of autophagy in M-MDSC-mediated suppression of antitumor immune responses. We demonstrate that autophagy-deficient M-MDSCs are reprogrammed, since they lose their suppressive activity and promote antitumor immune responses. Absence of autophagy deregulates the lysosomal function of M-MDSCs that entails insufficient lysosomal degradation and subsequently elevated surface expression of MHC class II. Overall, these findings propose that manipulation of the autophagy pathway in MDSCs could be considered an immunotherapeutic protocol in cancer.

\section{Results}

Increased autophagy in MDSCs of patients with melanoma and melanoma-bearing mice. MDSC autophagy was first assessed in sorted highly pure HLA-DR ${ }^{\mathrm{lo} /-} \mathrm{CD} 14^{-} \mathrm{CD} 33^{+} \mathrm{CD} 15^{+}$MDSCs isolated from peripheral blood of patients with melanoma (stage III-IV) and healthy controls (Figure 1A), following the updated guidelines for autophagy (20). Using immunofluorescence microscopy, we determined formation of functional autophagolysosomes based on the expression of LC3 that denotes formation of autophagosomes $(20,21)$, the lysosomal-associated membrane protein 1 (LAMP-1), and the adaptor protein SQSTM1/p62 that targets ubiquitinated proteins for lysosomal degradation (22). Notably, increased formation of autophagosomes was demonstrated in MDSCs from patients with melanoma compared with MDSCs from healthy individuals, and colocalization analysis revealed increased puncta positive for both p62 and LC3, indicating operation of functional autophagy (Figure 1B). Next we examined MDSC autophagy levels in a clinically relevant melanoma mouse model that entails subcutaneous injection of B16-F10 melanoma cells into C57/ BL6 mice. Upon melanoma establishment, CD11c ${ }^{-} \mathrm{CD} 11 \mathrm{~b}^{+} \mathrm{Gr} 1^{+}$ MDSCs were significantly enriched in the spleen of tumor-bearing mice, whereas the frequencies of the monocytic and granulocytic MDSC subsets were not altered (Figure 2A). Furthermore, assessment of autophagy in sorted MDSCs indicated increased levels of autophagosome formation and decreased levels of $\mathrm{p} 62$ compared with naive animals (Figure 2B). Importantly, MDSCs from tumors also demonstrated a prominent autophagolysomal function based on LC3 and p62 expression (Figure 2B).

The kinase mTOR-dependent pathway is the best-characterized regulator of autophagy, and activation of the PI3K/Akt axis is an upstream modulator of mTOR activity (23). To this end, we observed decreased phosphorylation of AKT (pAKT), mTOR (pmTOR), and the ribosomal protein S6 (pS6) in MDSCs from melanoma-bearing mice compared with naive controls (Figure 2C). In addition, phosphorylation of serine/threonine kinase UNC-51-like kinase 1 (ULK-1), which is required for activation of the preinitiation complex in the canonical pathway of autophagy $(24,25)$, was significantly increased in MDSCs from spleen and tumors of melanoma mice (Figure 2D). Collectively, these findings demonstrate a substantial upregulation and completion of the autophagy pathway in MDSCs from patients with melanoma and melanoma-bearing mice.

Because MDSCs comprise a heterogeneous population of monocytic and granulocytic progenitors (4), we sought to determine how the autophagy pathway is regulated in the respective MDSC subsets. To this end, both subsets exhibited enhanced autophagy as demonstrated by the increase in LC3 and the decrease in p62 puncta formation (Supplemental Figure 1; supplemental material available online with this article; https://doi. org/10.1172/JCI120888DS1).

Attenuated tumor growth and induction of potent antitumor immune responses in mice deficient for autophagy in the myeloid compartment. Next, we assessed whether autophagy possesses a functional role in MDSC-mediated tumor immune evasion. We generated LysM $^{\text {cre }} A \operatorname{Atg} 5^{\mathrm{fl} / \mathrm{fl}}$ mice (hereafter denoted as $A \operatorname{tg} 5^{4 L y s M}$ ) that lack Atg5 expression, an essential autophagy component, in the myeloid compartment. qPCR and Western blot analysis confirmed the marked reduction of Atg5 expression in MDSCs (Supplemental Figure 2, A and B) but not in T cells, whereas its expression in $\mathrm{CD} 11 \mathrm{c}^{+}$DCs was $50 \%$ reduced in $\operatorname{Atg} 5^{\triangle L y s M}$ mice compared with control littermates (Supplemental Figure 2A). In addition, Atg $5^{4 L y s M}$ mice did not show any alterations in the frequencies of $\mathrm{CD}^{+} \mathrm{T}$ cells, $\mathrm{CD}^{+} \mathrm{T}$ cells, and Foxp3 ${ }^{+}$Tregs either in the thymus or in the lymph nodes (LNs) (Supplemental Figure 2, C and D). Interestingly, B16-F10 melanoma growth was significantly attenuated in $\operatorname{Atg} 5^{4 L y s M}$ mice as compared with control Atg $5^{f / f l}$ mice (Figure 3A). This was not restricted to melanoma cells, since significant inhibition of Lewis lung carcinoma (LLC) cell growth was observed in $\operatorname{Atg} 5^{\Delta L y s M}$ mice compared with $\operatorname{Atg} 5^{f / f l}$ littermates (Figure 3B). Analysis of tumor-draining LNs (tdLNs) revealed no differences in the frequencies of $\mathrm{CD}^{+}$and $\mathrm{CD} 8^{+} \mathrm{T}$ cells, whereas Foxp $^{+}$Tregs were significantly reduced in melanoma-bearing Atg $5^{1 L y s M}$ mice (Figure 3C). However, analysis of tumor-infiltrating cells demonstrated significantly increased frequencies of CD $45^{+}$ cells and $\mathrm{CD}^{+}$lymphocytes in $A t g 5^{4 L y s M}$ mice, whereas the levels of tumor-infiltrating Foxp $3^{+}$Tregs were markedly decreased compared with control animals (Figure 3D). In addition, the expression of Foxp3 CTLA4 and CD73 was not altered on Tregs, whereas expression of GITR was significantly decreased (Supplemental Figure 3). Immunohistological analysis of tumors confirmed the increased frequencies of CD $4^{+}$cells in $A t g 5^{4 L y s M}$ mice (Figure 3E). In addition, the frequencies of NK1.1 $1^{+}$cells significantly decreased in the tumors of $\operatorname{Atg} 5^{\Lambda L y s M}$ mice (Figure 3F). Importantly, $\mathrm{CD}^{+}$and NK1.1 $1^{+}$infiltrating cells in $\operatorname{Atg} 5^{\triangle L y s M}$ mice expressed significantly increased levels of IFN- $\gamma$ compared with control animals (Figure $3, \mathrm{~F}$ and $\mathrm{G})$. Overall, these findings demonstrate that absence of autophagy in the myeloid compartment elicits a robust antitumor immune response that attenuates tumor growth.

Tumor-derived autophagy-deficient M-MDSCs highly accumulate into tumors and exhibit diminished suppressive activity. We next asked how the MDSC compartment was affected during tumor development in $\operatorname{Atg} 5^{4 L y s M}$ mice. We observed increased frequencies of $\mathrm{CD} 11 \mathrm{~b}^{+} \mathrm{Gr} 1^{+}$MDSCs in the spleens of tumor-inoculated Atg $5^{\triangle L y s M}$ mice compared with control animals, whereas frequencies of CD11 $\mathrm{c}^{+}$DCs were not altered (Figure 4A). This was reflected by an increased accumulation of G-MDSCs and decreased levels of M-MDSCs (Figure 4B). Surprisingly, we found markedly increased frequencies of total MDSCs in tumors of $\operatorname{Atg} 5^{\perp L y s M}$ mice (Figure 4C), with a prominent increase in M-MDSCs, whereas frequencies of G-MDSCs were not different (Figure 4D). Immunohistochemistry of tumor sections with CD206, a marker expressed by tumorassociated monocytes (26), confirmed the increased infiltration of myeloid cells in $\operatorname{Atg} 5^{4 L y s M}$ tumors (Figure 4E). In addition, we 
A
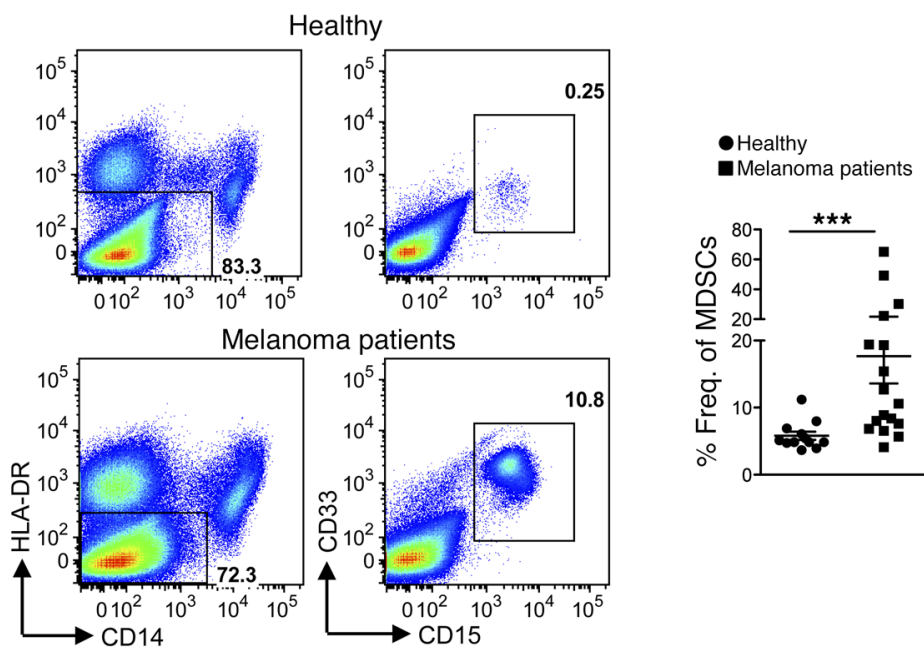

B
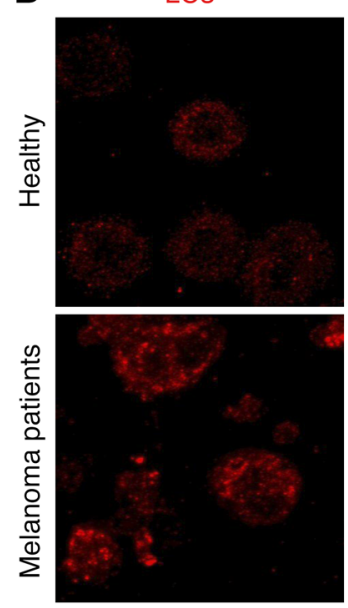

LAMP-1
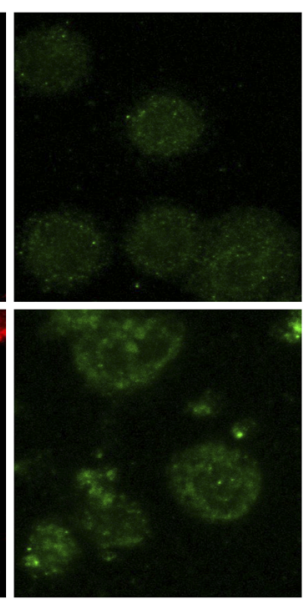
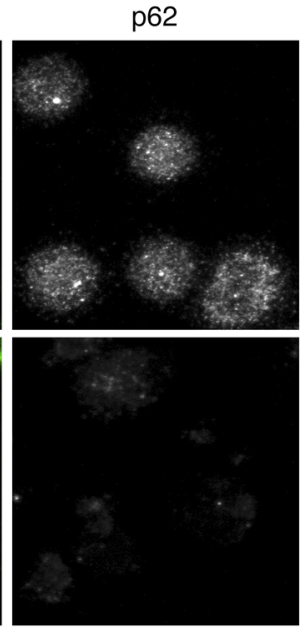

LC3/LAMP-1/p62/DAPI
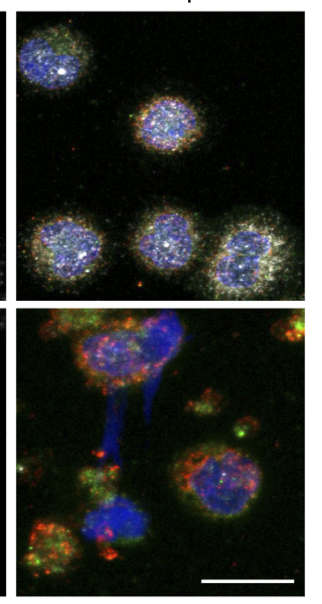

- Healthy

- Melanoma patients

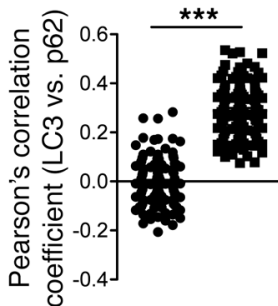

Figure 1. Enhanced autophagy in MDSCs from patients with melanoma. (A) Gating strategy and frequencies of MDSCs (HLA-DR-CD14-CD33+CD15+) in PBMCs of healthy individuals $(n=18)$ and patients with melanoma $\left.(n=17){ }^{* * *} P<0.0001\right)$. (B) Representative confocal microscopy images for LC3 (red), LAMP-1 (green), p62 (silver white), and DAPI (blue), and Pearson's correlation of LC3 versus $p 62\left({ }^{* * *} P<0.0001\right)$ in sorted MDSCs from peripheral blood of healthy individuals $(n=4)$ and patients with melanoma $(n=4)$. Scale bar: $10 \mu \mathrm{M}$. One representative experiment of 3 is shown. Results are mean \pm SEM. Statistical significance was obtained by unpaired Student's $t$ test.

found that all MDSCs expressed CD115 in the tumor milieu, and that CD40 expression, which has been linked to Treg induction by MDSCs (27), was significantly upregulated in $A \operatorname{tg} 5^{41 \mathrm{Ly} s \mathrm{M}}$ mice (Supplemental Figure 4A). Of interest, increased apoptosis was observed in tumor-infiltrating autophagy-deficient M-MDSCs (Supplemental Figure 4B), likely because of their increased turnover.

The augmented frequencies of M-MDSCs in the TME of Atg $5^{41 J^{3 s M}}$ mice along with attenuated tumor growth prompted us to examine the functional properties of autophagy-deficient M-MDSCs. Thus we sorted highly pure M-MDSCs from tumors of Atg5 $5^{41 / 5 s M}$ and control mice and examined their suppressive properties in vitro. To this end, M-MDSCs from $A \operatorname{tg} 5^{4 L y s \mathrm{~s} M}$ mice failed to suppress the proliferation as well as activation (based on CD44 expression) of $\mathrm{CD}^{+}{ }^{+} \mathrm{T}$ cells, in contrast to M-MDSCs isolated from control animals, which were highly suppressive in vitro (Figure 4 F). Importantly, coinjection of M-MDSCs from tumor-inoculated Atg $5^{41 / 5 s M}$ mice with B16-F10 cells resulted in significantly reduced tumor volume and weight (Figure 4G) and increased frequencies of $\mathrm{CD}^{+} \mathrm{T}$ cells in tdLNs compared with control M-MDSCs (Figure $4 \mathrm{H})$. Collectively, these data demonstrate that tumor-derived autophagy-deficient M-MDSCs exhibit diminished suppressive activity in vitro and in vivo.

Autophagy deficiency enhances the immunogenic properties of tumor-derived M-MDSCs through impaired lysosomal degradation of MHC II molecules. To elucidate the molecular mechanism through which autophagy dictates the suppressive activity of M-MDSCs, we performed whole-genome RNA sequencing of M-MDSCs isolated from tumor-inoculated $A \operatorname{tg} 5^{41 / 5 s M}$ and control animals and we found more than 1,300 genes to be differentially regulated (Figure 5A). Clustering and gene ontology analysis pointed to an enrichment of genes encoding molecules that belong to the lysosomal compartment (Figure 5B). In support of this, flow cytometric analysis of LysoSensor Green (DND-189), a weak base that accumulates in acidic organelles and which fluorescence is increased upon proton- 
A

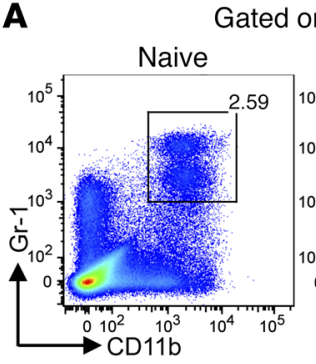

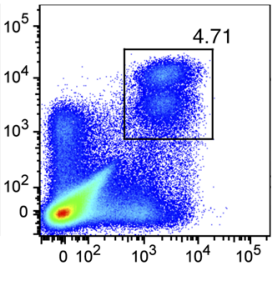

- Naive

- B16-F10

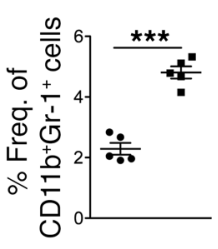

Gated on CD11 bi

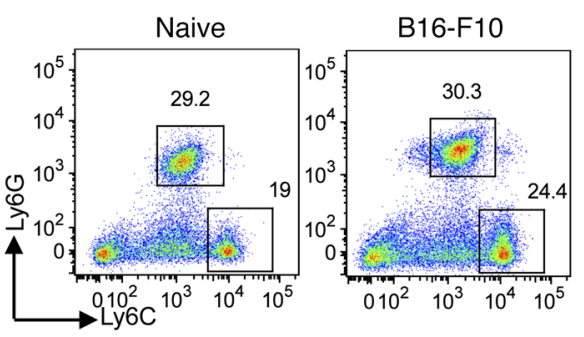

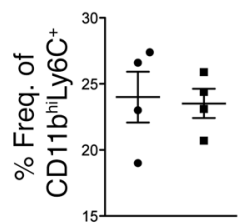

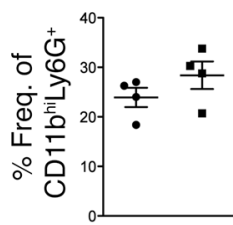

B
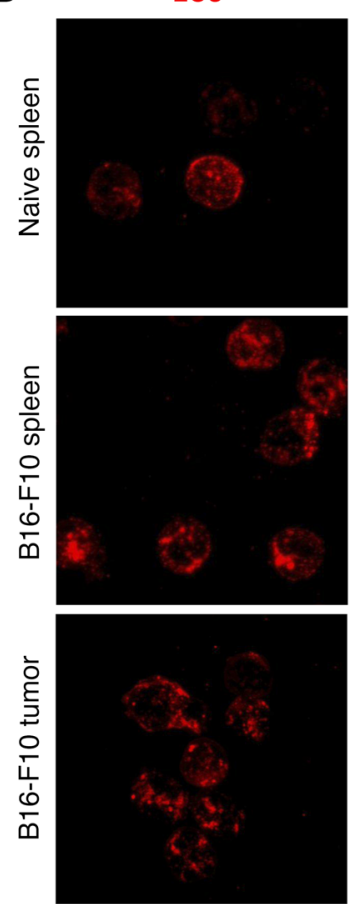

LAMP-1
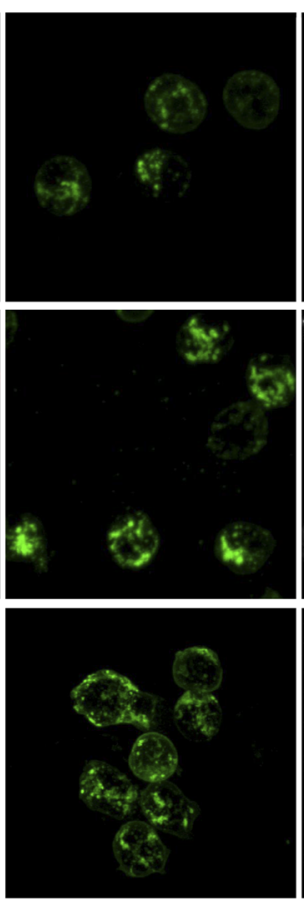

p62
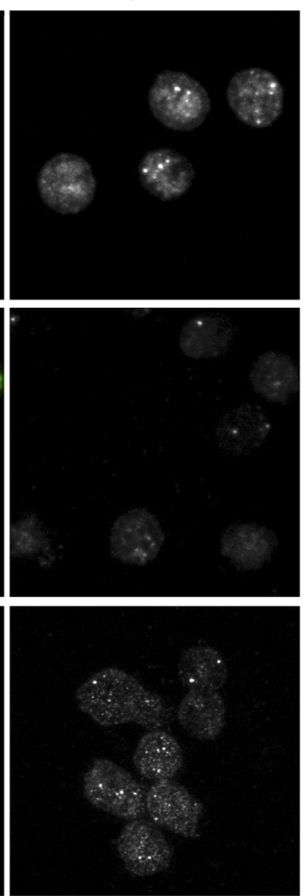

LC3/LAMP-1/p62/DAPI
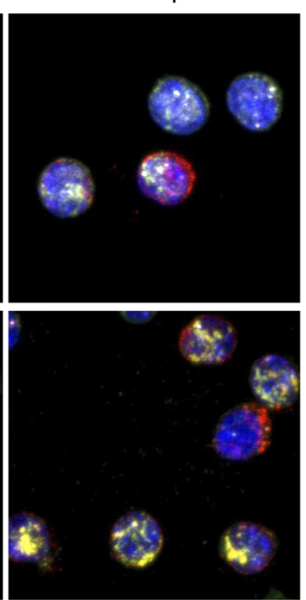

- Naive spleen

- B16-F10 spleen

A B16-F10 tumor
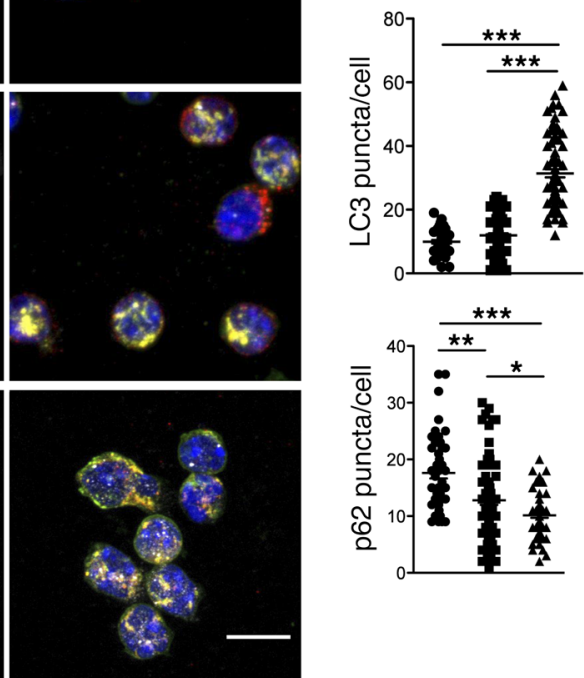

C

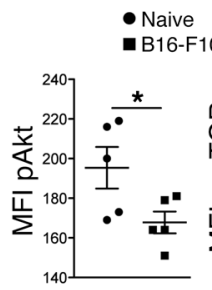

D
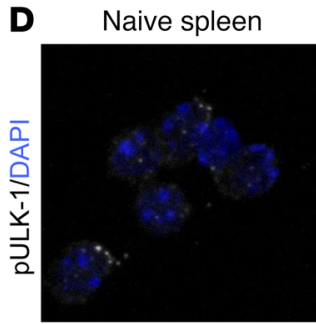

B16-F10 spleen

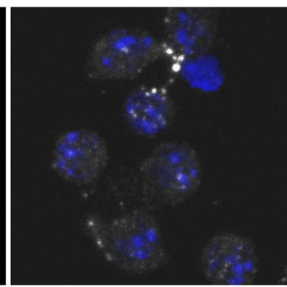

B16-F10 tumor

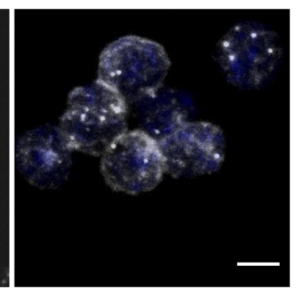

- Naive spleen B16-F10 spleen - B16-F10 tumor

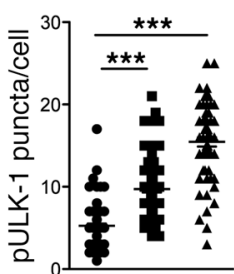

Figure 2. Upregulation of the autophagy pathway in MDSCs from melanoma-bearing mice. (A) Representative flow cytometric analysis and frequencies of total MDSCs (CD11c-CD11 $\left.\mathrm{b}^{+} \mathrm{Gr}-1^{+}\right)\left(n=5\right.$ mice per group, $\left.{ }^{* *} P<0.0001\right)$ and subsets from spleens of naive or B16-F10-inoculated mice $(n=4)$. (B) Representative immunofluorescence confocal images for LC3 (red), LAMP-1 (green), p62 (silver white), and DAPI (blue), and LC3 puncta/cell and p62 puncta/ cell in sorted MDSCs from spleens and tumors of naive and B16-F10-inoculated mice ( $n=4$ mice per group) (LC3: ${ }^{* * *} P<0.0001 ; p 62:{ }^{*} P=0.0459,{ }^{* *} P=$ 0.0003 , $\left.{ }^{* * *} P<0.0001\right)$. Scale bars: $10 \mu \mathrm{m}$. (C) MFI of pAkt $\left({ }^{*} P=0.0483\right)$, pmTOR $\left({ }^{*} P=0.0515\right)$, and pS6 $\left({ }^{* *} P=0.0027\right)$ in MDSC from spleens of naive or B16-F10 inoculated mice, $n=5$ mice per group. (D) Representative immunofluorescence confocal images for pULK-1 (silver white), and DAPI (blue), and pULK-1 puncta/cell in sorted MDSCs from spleens and tumors of naive and B16-F10-inoculated mice (pULK- ${ }^{* * *} P<0.0001$ ). Scale bar: $10 \mu$ m; $n=5$ mice per group. One representative experiment of 3 is shown. Results are mean \pm SEM. Statistical significance was obtained by unpaired Student's $t$ test (A and C) or 2-way ANOVA (B and D).

ation (28), revealed increased mean fluorescence intensity (MFI) in M-MDSCs from spleen and tumor of $\operatorname{Atg} 5^{4 L y s M}$ mice (Figure 5C). Furthermore, we assessed the expression of Rab7 GTPase, which is known to regulate intracellular membrane trafficking of endo- somal/lysosomal compartments (29), and expression of cathepsin D (cathD) in M-MDSCs isolated from $\operatorname{Atg} 5^{4 L y s M}$ and $\operatorname{Atg} 5^{f / f l}$ mice. Although expression of Rab7 was significantly decreased, the number of LAMP-1 puncta was not different in Atg $5^{4 L y s M}$ tumor- 
A

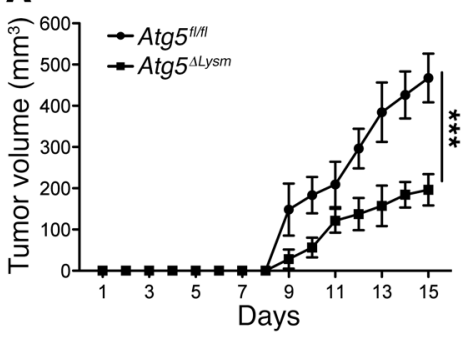

D
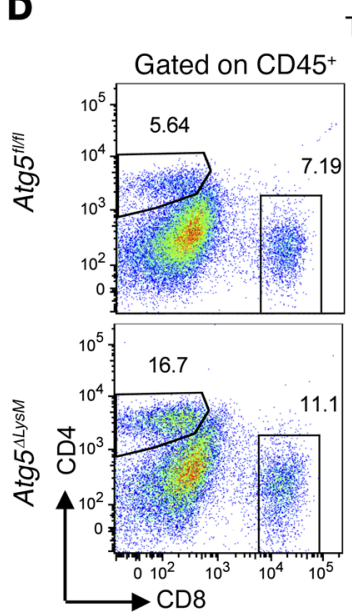

Tumor
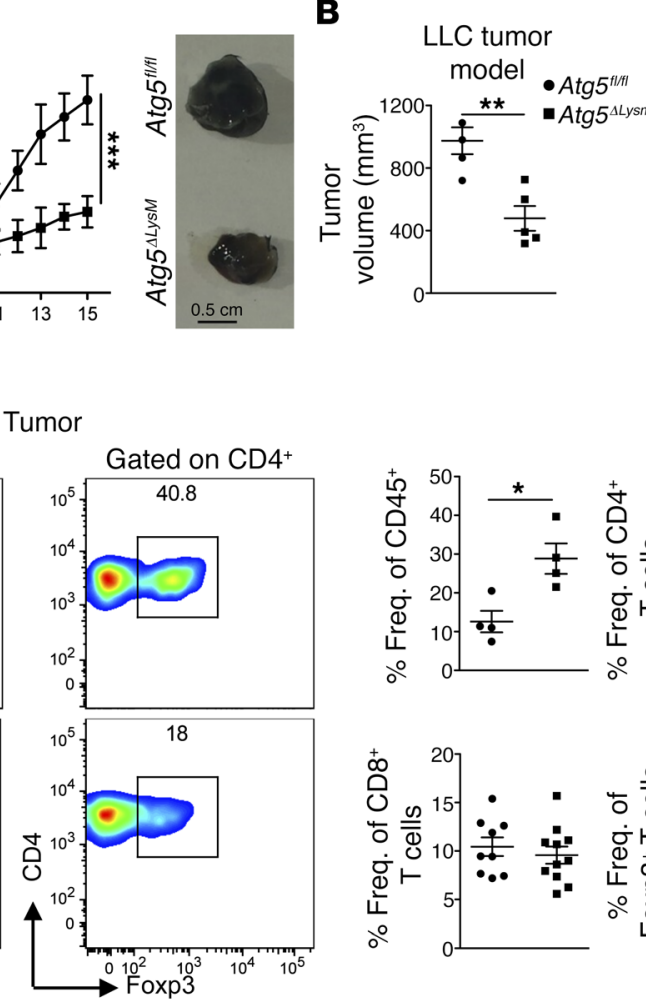

C

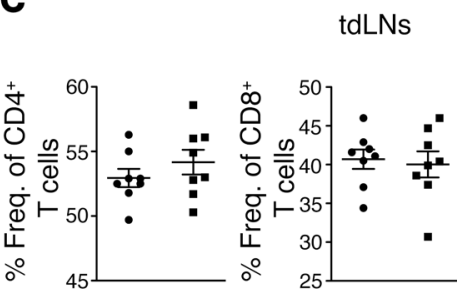

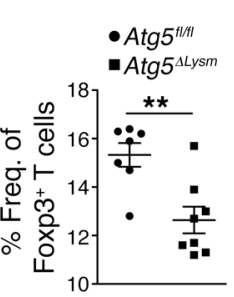

$\mathbf{F}$

\section{Gated on CD45 ${ }^{+}$}
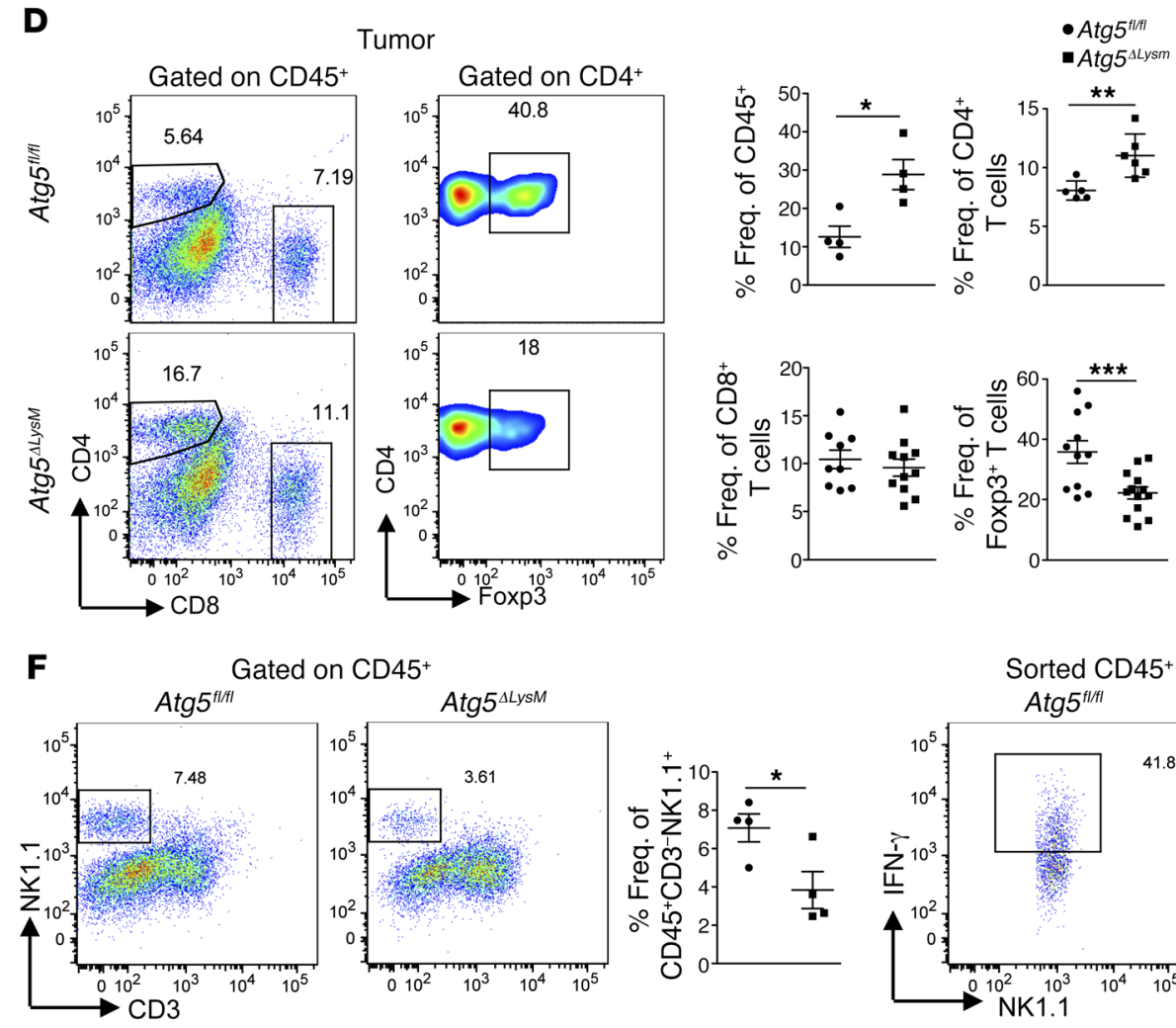

\section{E}
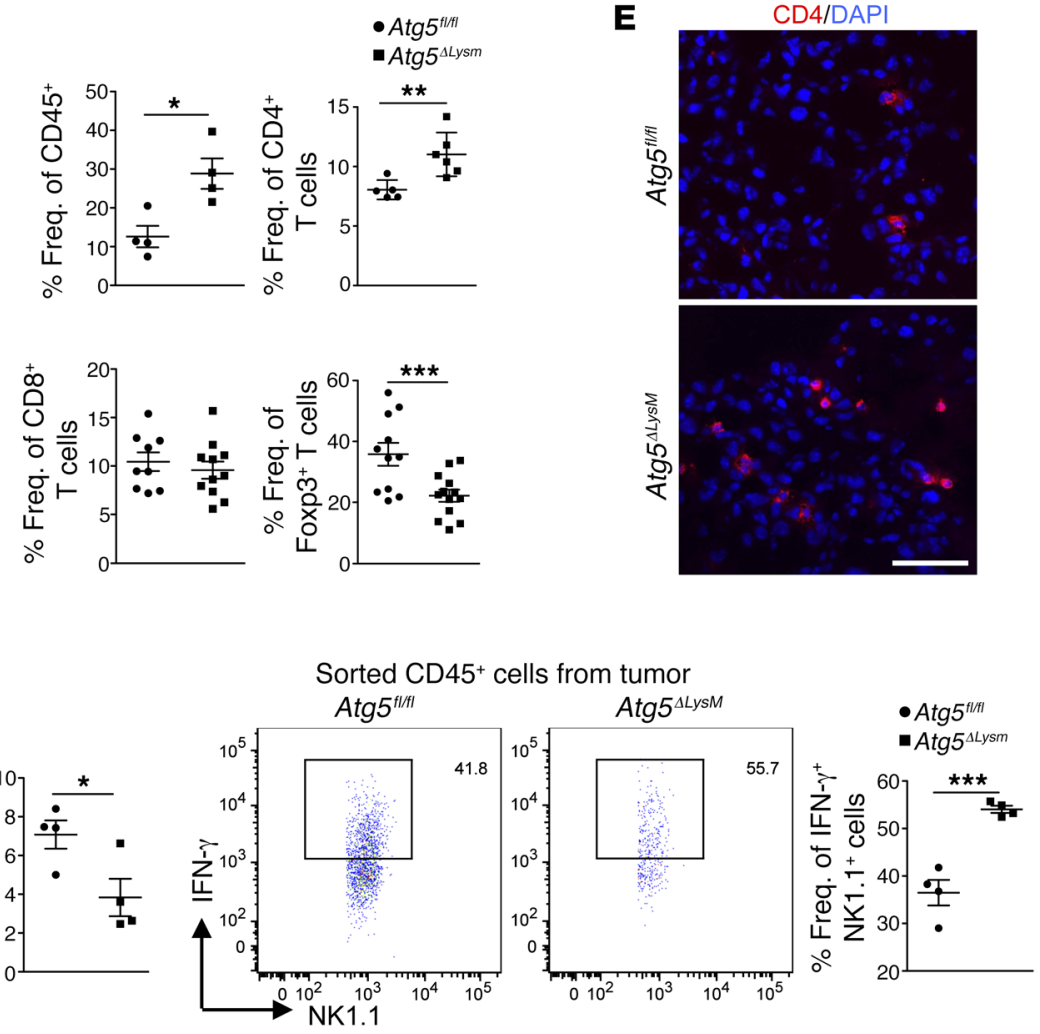

G

Tumor gated on CD8+
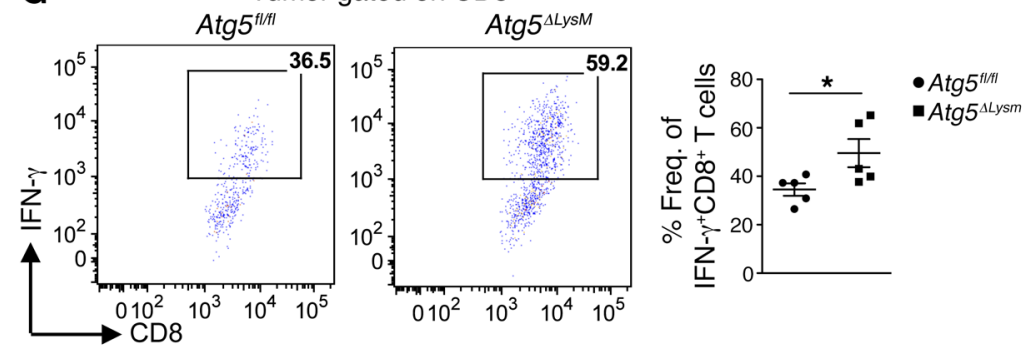

Figure 3. Deficiency of autophagy pathway in the myeloid compartment attenuates tumor growth and enhances antitumor immune responses. (A) Tumor volume $\left.{ }^{* * *} P=0.0005\right)$ and representative image of excised tumors of B16-F10-inoculated $A \operatorname{tg} 5^{\Delta L \nu s M}$ and $A \operatorname{tg} 5^{f l / f l}$ control mice. Representative results from 3 independent experiments are shown, $n=5$ mice per group. (B) Tumor volume ( $\left.{ }^{* *} P=0.0028\right)$ of LLC-inoculated $A \operatorname{tg} 5^{\Delta L / 4 s M}(n=4)$ and $A \operatorname{tg} 5^{f / / f l}$ control $(n=5)$ mice. Representative results from 3 independent experiments are shown. (C) Frequencies of CD4+, CD8 ${ }^{+}$T cells, and CD4 ${ }^{+}$Foxp3 ${ }^{+}$Tregs $\left(^{* *} P=\right.$ $0.0032)$ in tdLNs of B16-F10-inoculated $A \operatorname{tg} 5^{4 L y s M}$ and $A \operatorname{tg} 5^{f / / f l}$ control mice, $n=8$ mice per group. (D) Gating strategy and frequencies of CD45 ${ }^{*} P=0.0150$, $\left.n=4), \mathrm{CD}^{+}{ }^{(* *} P=0.0088, n=6\right), \mathrm{CD}^{+}(n=10)$, and CD4 ${ }^{+}$Foxp3 ${ }^{+}$Tregs $\left({ }^{* * *} P=0.0030, n=11\right)$, in tumor sites of Atg $5^{4 L y s M}$ and Atg $5^{f l / f l}$ control mice. For C and D, representative results from 4 independent experiments are shown. (E) Representative digital slide scanner images of CD4 ${ }^{+}$T cells (red) and DAPI (blue) in tumor section from B16-F10-inoculated $A \operatorname{tg} 5^{4 L y s M}$ and $A t g 5^{f l / f l}$ control mice are shown. Scale bar: $50 \mu \mathrm{m} ; n=5$ mice per group. (F) Representative flow cytometric analysis and frequencies of NK cells $\left({ }^{*} P=0.0364\right)$ gated on $\mathrm{CD}^{+} 5^{+}$and CD3-NK1.1+IFN- $\gamma^{+}$NK cells $\left({ }^{* * *} P=0.0008\right)$ in tumor site, $n=4$ mice per group. (C) Gating strategy and frequencies of CD8 ${ }^{+} I F N-\gamma^{+}$T cells $\left({ }^{*} P=0.0456\right)$ in tumor site. Representative results from 4 independent experiments are shown, $n=5$ mice per group. Results are mean \pm SEM. Statistical significance was obtained by unpaired Student's $t$ test. 
inoculated animals. Importantly, the expression of cathD was also significantly decreased (Supplemental Figure 5). Finally, we examined lysosomal function by using a long-lived degradation assay and pulsing M-MDSCs isolated from Atg $5^{4 L y s M}$ and Atg $5^{f / f l}$ mice with $\left[{ }^{3} \mathrm{H}\right]$ leucine, which further confirmed the impaired degradation capacity of lysosomes in autophagy-deficient M-MDSCs (Figure 5D). Since in tumor-bearing Atg5 $5^{4 L y s M}$ mice we observed a prominent increase in $\mathrm{CD}^{+} \mathrm{T}$ cell responses and the lysosomal compartment has been linked to the regulation of MHC II turnover (30), we questioned whether autophagy-deficient M-MDSCs have increased levels of MHC II surface expression due to impaired lysosomal degradation. To this end, flow cytometric analysis demonstrated a significant increase of surface MHC II levels in M-MDSCs from both spleen and tumors of $A \operatorname{tg} 5^{4 L y s M}$ mice (Figure 5E) as well as blood M-MDSCs (Supplemental Figure 6), whereas MHC II expression in DCs was not altered (Figure 5E). Interestingly, significantly increased levels of IA ${ }^{\mathrm{b}}$ colocalized with LAMP-1 in M-MDSCs from melanoma-bearing $\operatorname{Atg}^{5 L y s M}$ mice (Figure 5F), suggesting that $\mathrm{IA}^{\mathrm{b}}$ molecules do not efficiently degrade in the lysosomal compartment. Blocking of lysosomal function with $\mathrm{NH}_{4} \mathrm{Cl}$ or chloroquine in tumor explant supernatant-treated (TES-treated) M-MDSCs from B16-F10-inoculated mice demonstrated significantly increased surface expression of $\mathrm{IA}^{\mathrm{b}}$ molecules without affecting the transcription of IAb or Ciita (the master regulator of MHC II expression) genes (Figure 5G).

To examine the functional importance of increased $\mathrm{MHC}$ II expression in autophagy-deficient M-MDSCs, we cocultured OVA peptide-pulsed M-MDSCs with sorted CellTrace-labeled $\mathrm{CD}^{+} \mathrm{CD} 25^{-} \mathrm{V} \alpha 2^{+}$OTII cells. Autophagy-deficient M-MDSCs demonstrated a superior ability to induce OTII T cell proliferation in vitro compared with WT M-MDSCs (Figure 6A). In a similar fashion, adoptive transfer of $\mathrm{CD}^{+} \mathrm{CD} 25^{-} \mathrm{V} \alpha 2^{+}$OTII T cells into B16-F10-OVA.GFP-inoculated Atg $5^{\triangle L y s M}$ mice resulted in enhanced activation (based on CD25 and CD44 expression) of the transferred OTII T cells compared with those transferred in Atg $5^{f / f l}$ animals (Figure 6B), suggesting that autophagy-deficient M-MDSCs could efficiently present tumor-specific "neo-antigens," and this was greatly enhanced in autophagy-deficient M-MDSCs.

Finally, to provide direct evidence for the importance of the aberrant lysosomal degradation of MHC II molecules in the enhancement of antitumor immunity, we performed an siRNAmediated knockdown of March1 (Figure 6C), the membraneassociated RING-CH1 (MARCH1) E3 ubiquitin ligase, which mediates the MHC II internalization and trafficking to lysosomes (30) in M-MDSCs. Our results demonstrated a significant increase in the surface expression of $\mathrm{IA}^{\mathrm{b}}$ molecules in tumor-derived M-MDSCs transfected with March1 siRNA- compared with control siRNA-transfected cells (Figure 6D). Importantly, adoptive transfer of March1-silenced M-MDSCs markedly reduced melanoma growth (Figure 6E) and induced a robust antitumor immune response (Figure 6F). Overall, these findings provide evidence for an important role of autophagy in dictating the suppressive program of M-MDSCs through regulation of lysosomal degradation of MHC class II molecules.

\section{Discussion}

Herein we provide evidence for a crucial role of the autophagy pathway in the function of tumor-associated MDSCs. In the absence of autophagy, M-MDSCs are reprogrammed to highly immunogenic cells eliciting an endowed antitumor immune response that promotes tumor eradication. Mechanistically, transcriptomic and functional analysis demonstrated an aberrant lysosomal degradation of MHC II in autophagy-deficient M-MDSCs that contributed to its increased surface expression and resulted in more efficient priming of antitumor $\mathrm{CD} 4^{+} \mathrm{T}$ cell responses.

The effectiveness of checkpoint blockade immunotherapies is limited to patients with a high proportion of tumor-infiltrating lymphocytes (TILs), and major efforts have been made toward enhancing the recruitment of TILs into the TEM to induce potent antitumor immune responses (31). In addition, tumor-reactive effector $\mathrm{CD} 4{ }^{+} \mathrm{T}$ cells have been shown to enhance and sustain the accumulation of $\mathrm{CD}^{+}$cytotoxic T cells within tumors $(32,33)$, and tumor-specific Th17 cells potently inhibit melanoma growth (34) via intratumoral recruitment of DCs and development of potent antitumor cytotoxic $\mathrm{T}$ cell responses (35). Finally, adoptive transfer of tumor-specific $\mathrm{CD} 4^{+} \mathrm{T}$ cells into tumor-bearing lymphopenic hosts resulted in complete tumor eradication mediated by potent cytotoxic activity of the $\mathrm{CD} 4^{+} \mathrm{T}$ cells (36). A prerequisite for efficient activation of tumor-specific T cells is presentation of tumorassociated antigens (TAAs) by APCs in the context of MHC molecules and costimulation. MDSCs have been reported to express low levels of MHC in various tumor mouse models (37) and also in patients with tumors (38-40). One hypothesis is that low levels of MHC II expression by MDSCs would render them tolerogenic, which would preferentially promote induction of Tregs. In support of this hypothesis, in a B-cell lymphoma mouse model, MDSCs expressing low levels of MHC II served as tolerogenic APCs, engulfed and presented tumor antigens, and induced Tregmediated tolerance (41). Furthermore, CD14 ${ }^{+} \mathrm{HLA}^{-D R^{-/ 10}}$ MDSCs isolated from patients with hepatocellular carcinoma induced $\mathrm{CD}^{+}{ }^{+} \mathrm{CD} 25^{+}$Foxp $^{+}$Tregs in vitro upon culture with autologous T cells (42); however, a direct link with HLA-DR expression was not shown. Our results demonstrate that autophagy sustains the low levels of MHC II surface expression in M-MDSCs, and thus contributes to their tolerogenic phenotype. Specifically, tumorexposed autophagy-deficient M-MDSCs showed impaired lysosomal degradation accompanied by marked surface expression of MHC II molecules and enhanced proliferation of tumor-derived antigen-specific $\mathrm{CD} 4^{+} \mathrm{T}$ cells in vivo. In addition, we observed increased accumulation of $\mathrm{IA}^{\mathrm{b}}$ molecules in the lysosomes of M-MDSCs from tumor-inoculated $\operatorname{Atg} 5^{\triangle L y s s}$ mice, suggesting an incomplete degradation of MHC II. Finally, in vitro blocking of lysosomal function substantially increased MHC class II surface expression without affecting $I A^{b}$ or Ciita gene expression. All the above pointed toward an autophagy-dependent aberrant lysosomal degradation of MHC II molecules in tumor-associated M-MDSCs. In line with our results, earlier studies showed that DCs with limited lysosomal proteolysis are more potent APCs than macrophages with high proteolytic capacity (43). To date, lysosomal degradation of MHC II molecules is considered to play an instrumental role in determining the levels of surface MHC II expression (30, 44, 45). Specifically, MHC II surface expression is regulated by the E3 ubiquitin ligase membrane-associated RINGCH1 (MARCH1), which mediates intracellular localization and lysosomal degradation of pMHC-II in DCs (46) and B cells (47). 
A
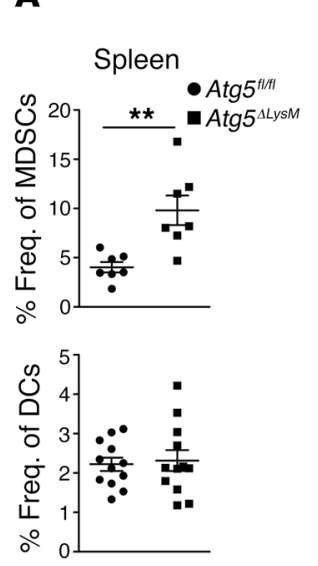

B

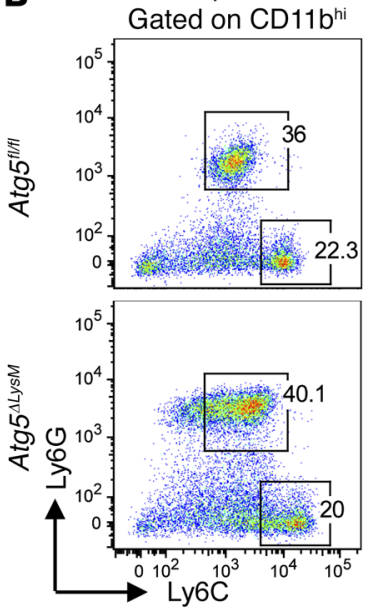

Spleen

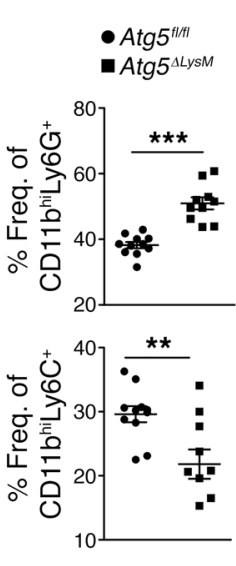

C

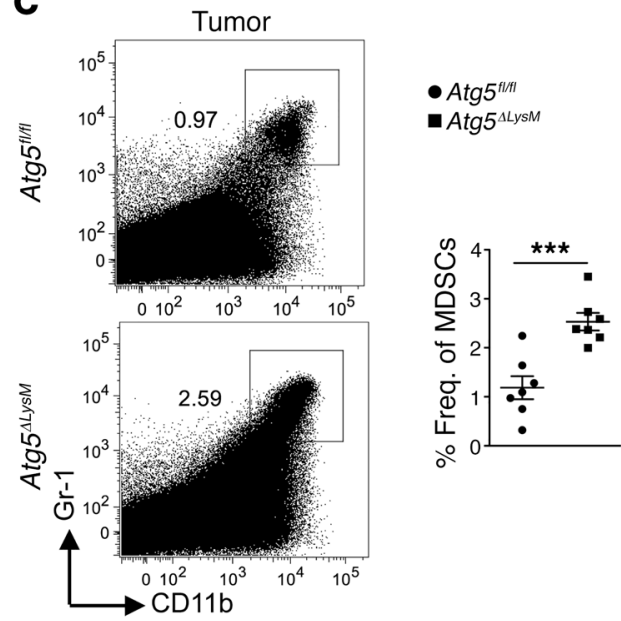

D

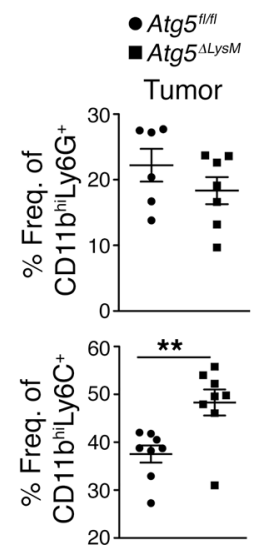

$\mathbf{E}$

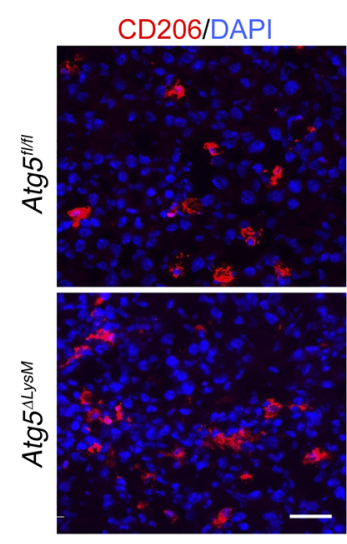

F

Gated on $\mathrm{CD}^{+} \mathrm{T}$ cells
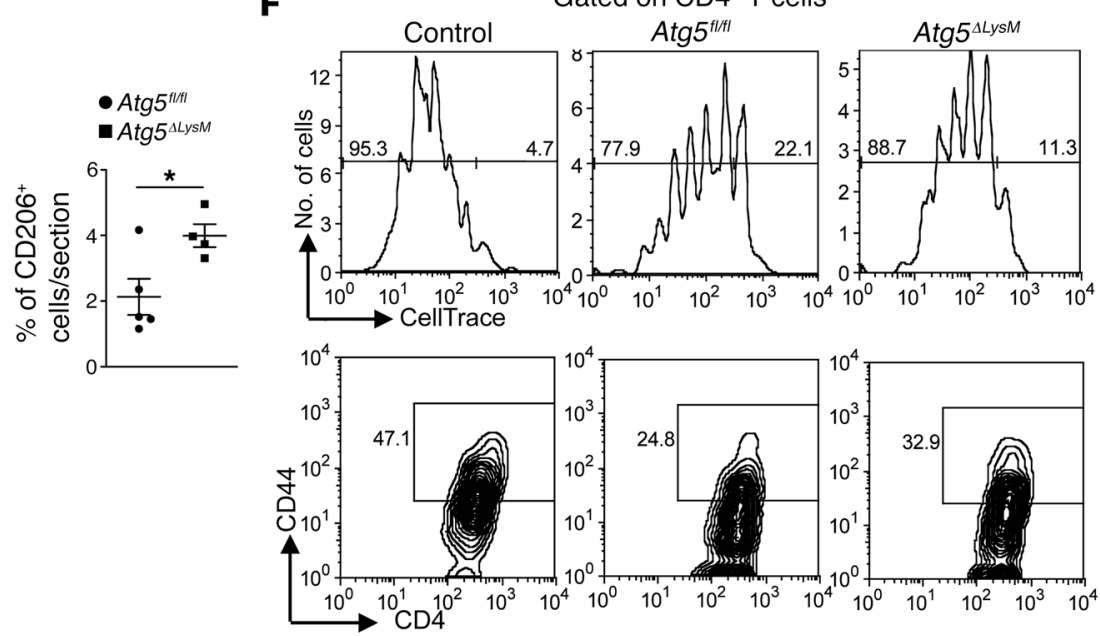

G
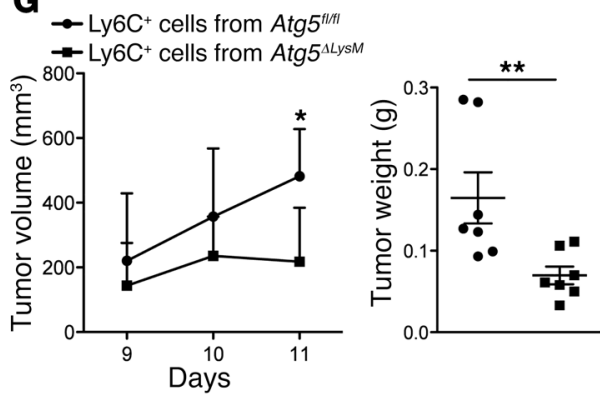

H

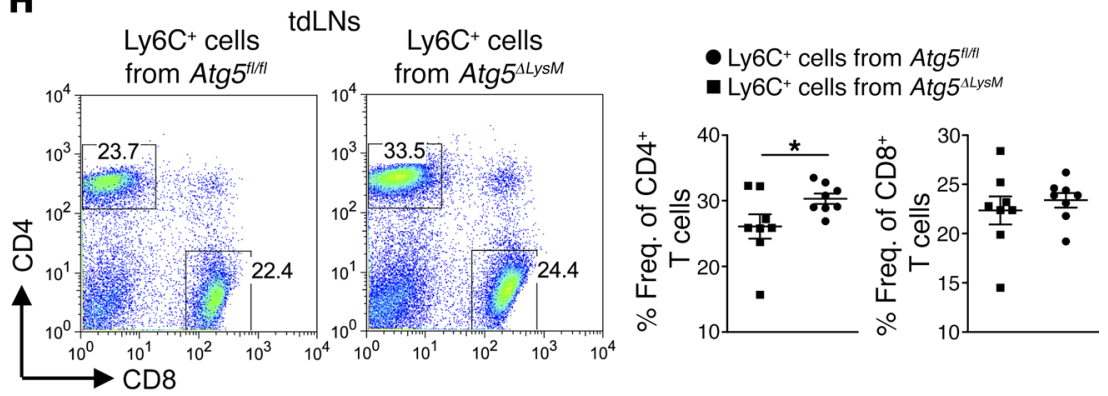

Figure 4. Impaired suppressive function of tumor-infiltrating autophagy-deficient M-MDSCs from melanoma-bearing mice. (A) Frequencies of MDSCs

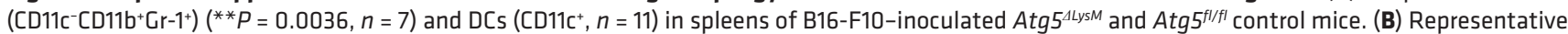

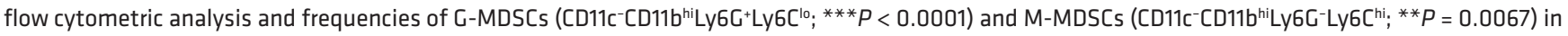
spleens of B16-F10-inoculated Atg $5^{L L / 4 S M}$ and $A t g 5^{f l / f l}$ control mice ( $n=10$ mice per group). (C) Representative flow cytometric analysis and frequencies of tumor-infiltrating MDSCs ( $\left.{ }^{* *} P=0.006\right)$ in B16-F10-inoculated Atg $5^{4 L y s M}$ and $A \operatorname{tg} 5^{f l / f l}$ control mice $(n=7$ mice per group). (D) Frequencies of G-MDSCs and M-MDSCs ( $\left.{ }^{* *} P=0.0050\right)$ in tumors of B16-F10-inoculated $A \operatorname{tg} 5^{A L y s M}$ and $A \operatorname{tg} 5^{f l / f l}$ control mice $(n=8$ mice per group). (E) Representative digital slide scanner images and percentages of $\mathrm{CD} 206^{+}$cells $\left({ }^{*} P=0.0310\right.$ ) (red) and DAPI (blue) per tumor section isolated from B16-F10-inoculated Atg $5^{\Delta L y s M}$ and Atg $5^{f l / f l}$ control mice. Scale bar: $40 \mu \mathrm{m} ; n=5$ mice per group. (F) Representative histograms of CD4 ${ }^{+} T$ cell proliferation and flow cytometric analysis of CD44 in CellTrace-labeled LNCs cultured with sorted M-MDSCs from tumors of Atg $5^{4 L y s M}$ and control Atg $5^{f / f f}$ B16-F10-inoculated mice, $n=4$ mice per group. For $\mathbf{G}$

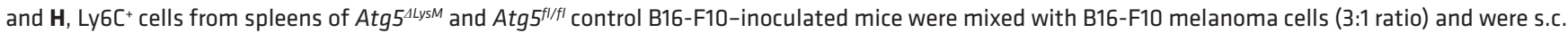
injected into C57BL/6 mice ( $n=8$ mice per group). (C) Tumor volume $\left({ }^{*} P=0.0082\right)$ and tumor weight $\left({ }^{* *} P=0.007\right)$ are shown. (H) Frequencies of CD4 ${ }^{+}$ ( $\left.{ }^{*} P=0.0499\right)$ and $C D 8^{+} T$ cells from tdLNs. Results are mean \pm SEM. Statistical significance was obtained by unpaired Student's $t$ test. Representative results from 3 independent experiments are shown. 
A

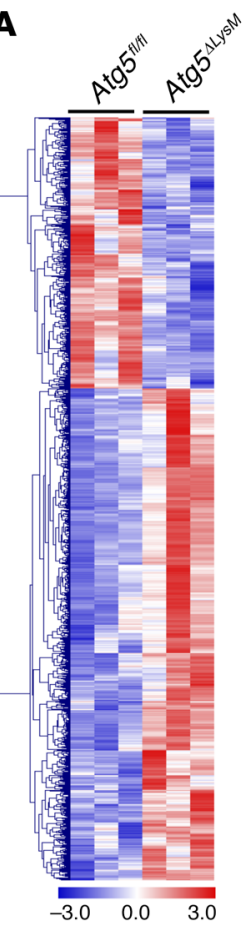

B

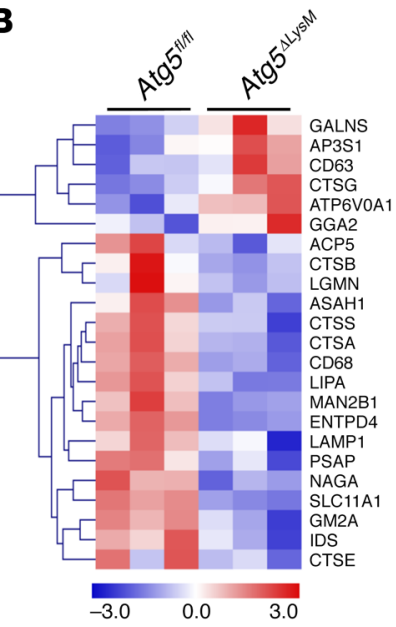

D

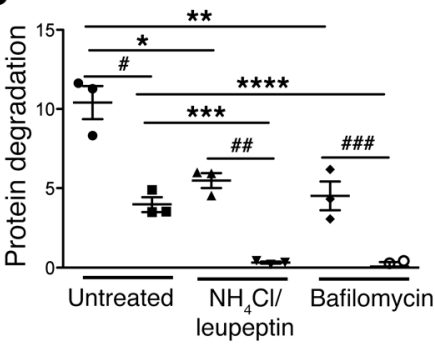

$\mathbf{F}$
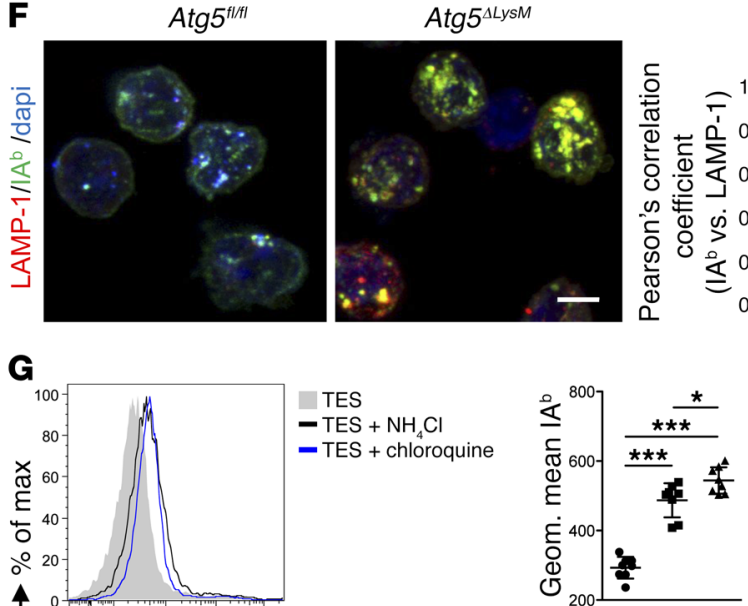

G

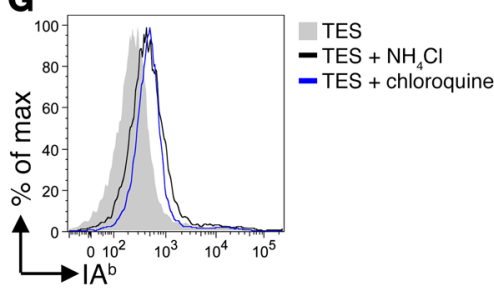

C
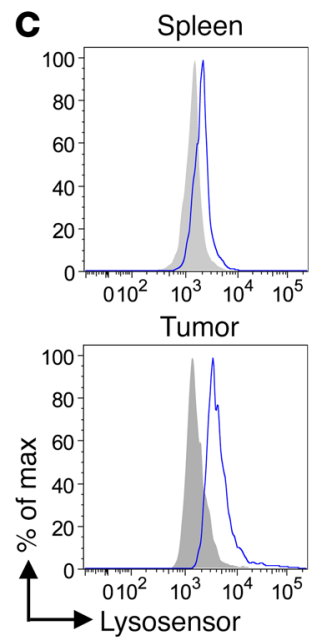
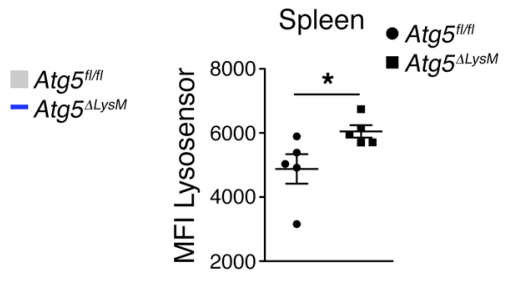

Tumor

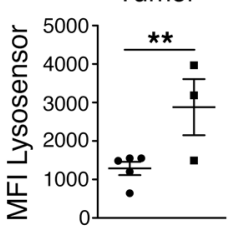

- $\operatorname{Atg} 5^{f / / f 1}$

- $\operatorname{Atg} 5^{\Lambda L y s M}$

$\Delta \operatorname{Atg} 5^{f|/| t \mid}$

Atg5 ${ }^{\text {LLysM }}$

$\leftrightarrow \operatorname{Atg} 5^{f|/| t \mid}$

- Atg5 $5^{\triangle L y s M}$

E

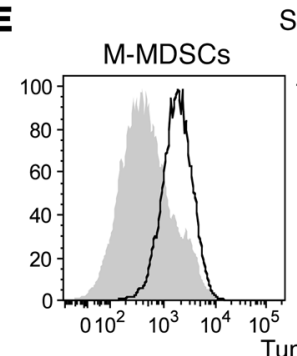

Spleen $\quad \operatorname{Atg}^{\| \prime \prime}$

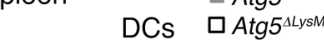

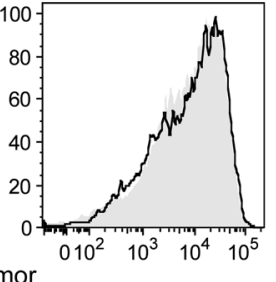

umor

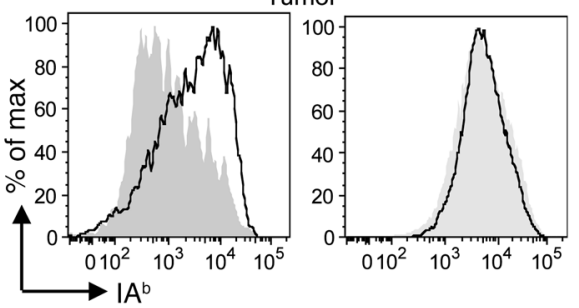

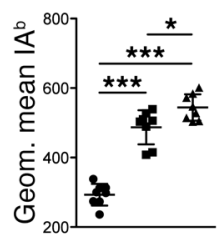

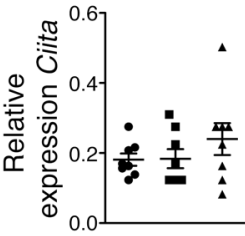

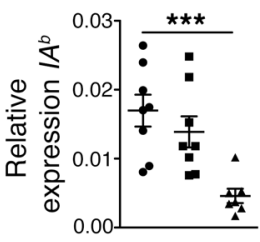

- TES

- TES + $\mathrm{NH}_{4} \mathrm{Cl}$

$\triangle \mathrm{TES}+$ chloroquine

Figure 5. Impaired lysosomal degradation and increased surface expression of MHC II molecules in autophagy-deficient M-MDSCs. (A) Heat map of differentially expressed genes in M-MDSCs isolated from spleens of B16-F10-inoculated Atg $5^{4 L y s M}$ and control mice ( $n=3$ mice per group). (B) Heat map of differentially expressed genes related to the lysosomal function in M-MDSCs isolated from the spleens of B16-F10 inoculated Atg $5^{4 L y s M}$ and control mice ( $n=$ 3 mice per group). (C) MFI of lysosensor in M-MDSCs from spleen ( $\left.{ }^{*} P=0.0470\right)$ and tumor $\left.{ }^{* *} P=0.0335\right)$ of B16-F10-inoculated $A$ tg $5^{\Lambda L y s M}$ and control mice ( $n=5$ mice per group). (D) Percentage of protein degradation, using [ $\left.{ }^{3} \mathrm{H}\right]$ leucine, in M-MDSCs isolated from the spleens of B16-F10 inoculated $A t g 5^{4 L 45 M}$ and control mice treated with lysosomal inhibitors ( $\mathrm{NH}_{4} \mathrm{Cl}$ and leupeptin or bafilomycin) or left untreated $\left(n=3\right.$ mice per group). ${ }^{*} P=0.0134,{ }^{*} P=0.0084$, ${ }^{* * *} P=0.0195,{ }^{* * *} P=0.0128,{ }^{*} P=0.0179,{ }^{* \#} P=0.0088,{ }^{* \#} P=0.0264$. (E) Representative histograms for the expression of IA ${ }^{\mathrm{b}}$ by M-MDSCs of spleen

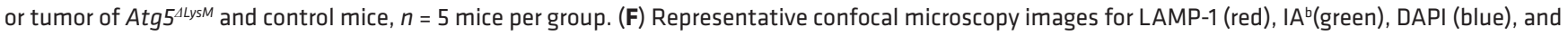
Pearson's correlation of IA ${ }^{b}$ versus LAMP-1 $\left({ }^{* * *} P<0.0001\right)$ in sorted M-MDSCs from splenocytes of B16-F10-inoculated Atg $5^{4 L y s M}$ and control mice $(n=4$ mice/group). Scale bar: $10 \mu \mathrm{m}$. (C) Representative histograms for the expression of IA by M-MDSCs isolated from spleens of B16-F10-inoculated Atg $5^{f / / f I}$ mice after in vitro stimulation with TES in the presence of $\mathrm{NH}_{4} \mathrm{Cl}$ or chloroquine. Geometric mean of $\mathrm{IA}{ }^{b}\left({ }^{* * *} P<0.0001,{ }^{*} P=0.048\right)$ and relative expression of Ciita and $I A^{b}\left({ }^{* * *} P<0.0001\right)$ are shown, $n=8$ mice per group. Results are mean \pm SEM. Statistical significance was obtained by unpaired Student's $t$ test, or 2-way ANOVA ( $\mathbf{D}$ and $\mathbf{G}$ ). Representative results from 3 independent experiments are shown. 

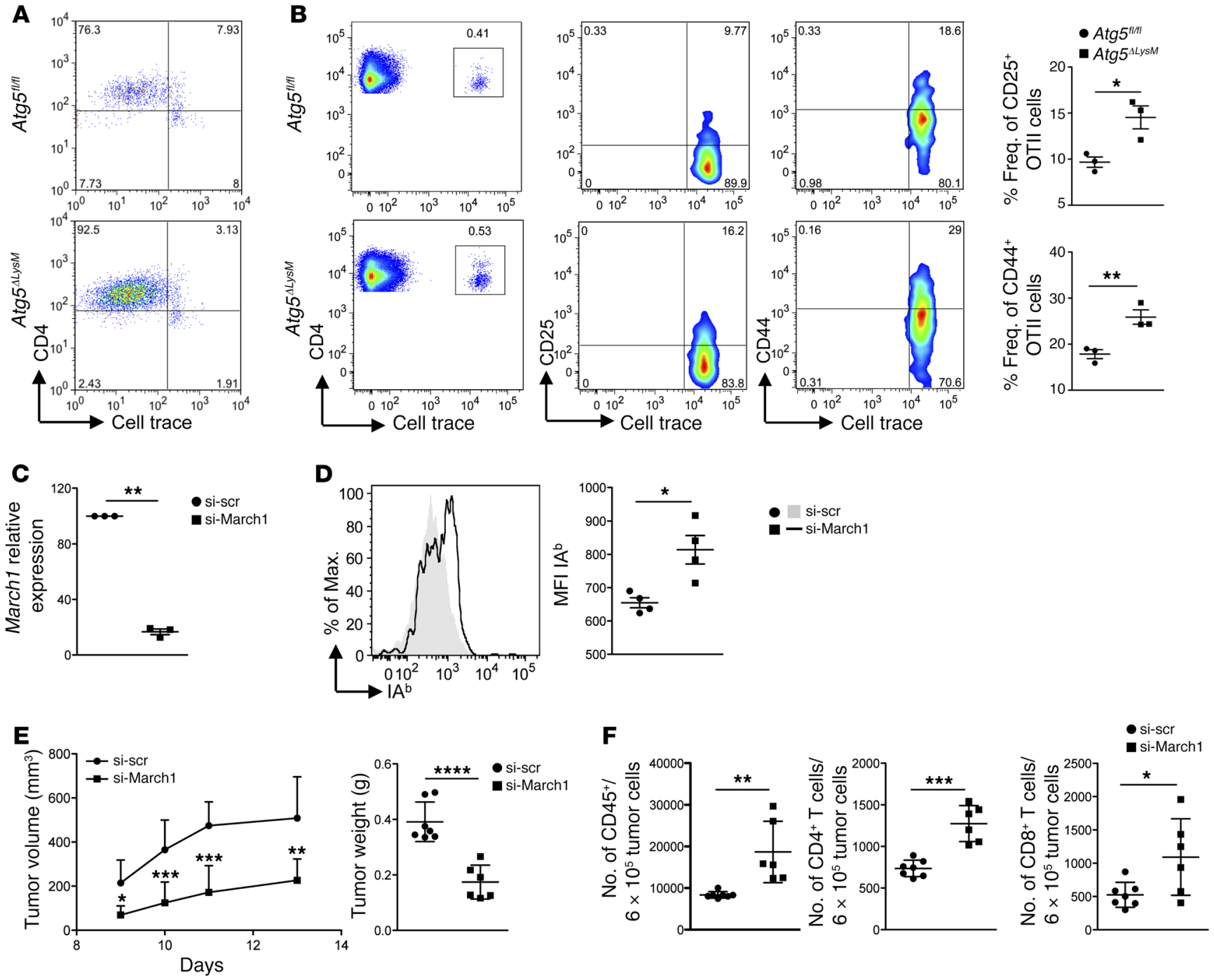

Figure 6. Sustained IA ${ }^{b}$ expression in autophagy-deficient tumor-derived M-MDSCs endows their immunogenic properties. (A) Representative flow cytometric analysis of CellTrace-labeled OTII CD4 ${ }^{+}$T cells cultured with M-MDSCs of Atg $5^{4 L y s M}$ and control B16-F10-inoculated mice in the presence of OVA peptide, $n=5$ mice per group. (B) Gating strategy and frequencies of $\operatorname{CD} 25^{+}\left({ }^{*} P=0.0236\right)$ and $\operatorname{CD} 44^{+}\left({ }^{* *} P=0.0116\right) 0$ TII CD4 ${ }^{+}$T cells adoptively transferred in $A \operatorname{tg} 5^{\Lambda L y s M}$ and control tumor-bearing mice, $n=3$ mice per group. (C) Relative March1 expression in M-MDSCs following transfection with siRNA for March1 or scramble si ( ${ }^{* *} P=0.0006, n=3$ mice per group). (D) Representative histograms and MFI for IA ${ }^{\mathrm{b}}$ expression $\left({ }^{*} P=0.0129\right)$ in M-MDSCs following transfection with siRNA for March1 or scramble si ( $n=4$ mice per group). For $\mathbf{E}$ and $\mathbf{F}, 4 \times 10^{5} \mathrm{M}$-MDSCs transfected with scramble si or si-March1 were s.c. coinjected with $3 \times 10^{5}$ B16-F10 cells in C57BL/6 mice ( $n=7$ mice per group). (E) Tumor volume $\left({ }^{*} P=0.0044,{ }^{*} P=0.017,{ }^{* *} P<0.0001\right)$ and tumor weight $\left({ }^{* * *} P<0.0001\right)$ are shown. $(\mathbf{F})$ Numbers of $\operatorname{CD} 45^{+}\left({ }^{* *} P=0.0035\right), \operatorname{CD} 4^{+}\left({ }^{* * *} P<0.0001\right)$ and $\operatorname{CD} 8^{+}\left({ }^{*} P=0.0307\right)$ T cells per $6 \times 10^{5}$ tumor cells are depicted. One representative experiment of 3 is shown. Results are mean \pm SEM. Statistical significance was obtained by unpaired Student's $t$ test.

To this end, DCs isolated from MARCH1-KO or MHC-II ubiquitination-mutant mice express very high levels of pMHC-II on the plasma membrane. In a similar fashion, our data provide additional evidence for the impaired lysosomal degradation of MHC II in determining the function of M-MDSCs, since silencing of March1 in M-MDSCs resulted in a marked expression of MHC II in the cell surface and, importantly, loss of M-MDSC suppressive activity, by promoting tumor rejection and enhancing antitumor immunity.

NK cell responses play an important role in antitumor immunity by exerting strong cytotoxic activity, and MDSCs have been shown to regulate their function not only in tumors but also in viral infections (48-50). Our findings show that the frequencies of NK1.1 $1^{+}$cells are decreased in the tumor and spleen of $\operatorname{Atg} 55^{41 / 2 s \mathrm{~s} M}$ mice, whereas the frequencies of intratumoral IFN- $\gamma$-producing NK cells are increased. Whether NK cell responses are regulated by M-MDSCs that dominate the tumor microenvironment in $\operatorname{Atg} 5^{4 L 5 s}$ s mice remains unknown. Indeed, only G-MDSCs have been closely linked to NK cell activity (48), suggesting that a thorough understanding of MDSC-mediated regulation of antitumor NK cell responses is required.

Our data demonstrate that the mTOR pathway is downregulated in MDSCs from tumor-inoculated mice, which is in agreement with increased autophagy levels. Although mTOR signaling regulates various physiological as well as pathological processes and has also been shown to affect autophagosome-lysosome fusion (51), its role in MDSC function just emerged. Specifically, it 
has been demonstrated that lysosomal acid lipase (lal) in MDSCs regulated their metabolic reprogramming and expansion mostly in granulocytic lineage and also that lat MDSCs exhibited increased activation of the mTOR signaling pathway (52). In a follow-up study, Rab7 GTPase was identified to regulate the mTOR in lal MDSCs and inhibition of Rab7 function substantially reduced MDSC differentiation as well as trans-endothelial migration (53). Our data show that impaired lysosomal function in autophagydeficient M-MDSCs is associated with decreased levels of Rab7, but had no effect on total LAMP-1 expression. Since MDSCs constitute a heterogenous population of monocytic and granulocytic cells that potently suppress antitumor immune responses, the delineation of signals that regulate the metabolic activities and are linked to lysosomal genesis and function in each subset are of great importance and warrant further investigation.

Given that MDSCs are the progenitors of granulocytes, macrophages, and DCs, delineation of the mechanisms involved in the MDSC differentiation are crucial for the development of therapeutic regimens that will benefit patients with cancer. Several methods have been developed to achieve this goal $(4,54,55)$; however, the clinical implementation of these approaches and their specificity have not been examined. Our findings propose targeting of autophagy as a novel pathway to alter M-MDSC suppressive activity. Indeed, whole-genome transcriptomic analysis of tumor-derived M-MDSCs provided evidence for a major impact of autophagy on their phenotype, which was confirmed with in vitro and in vivo functional assays. The autophagy pathway was found to be markedly upregulated in MDSCs from melanoma-bearing mice as well as patients with melanoma. Several factors could contribute to increased MDSC autophagy in tumors. Reduced oxygen supply is a cardinal feature of the TME and it has been linked to induction of autophagy (56), a process that is regulated through HIF-1 $\alpha$ (57). Expression of HIF-1 $\alpha$ has been closely linked to MDSC differentiation, since HIF- $1 \alpha$-deficient MDSCs fail to suppress and instead differentiate toward DCs and macrophages $(12,58)$. However, whether HIF-1 $\alpha$ deficiency in tumor-exposed MDSCs affects operation of autophagy was not addressed. Alternatively, under hypoxic conditions, M-MDSCs upregulated the expression of CD45 tyrosine phosphatase activity, which subsequently decreased STAT3 function and halted M-MDSC differentiation (59). Our results unravel autophagy as a new pathway whose regulation could be exploited to drive MDSC differentiation and impair its suppressive activity.

Collectively, autophagy has been shown to promote or suppress tumorigenic events in a highly context-dependent manner $(10,60)$, but its role in shaping the function of tumor-associated immune regulatory cells remains obscure. Our findings delineate a pivotal role of autophagy in redirecting the differentiation of M-MDSCs to immunogenic cells that promote tumor rejection. Manipulating autophagy or the molecular events that lead to enhanced autophagy in MDSCs therapeutically could offer new opportunities to enhance activation and expansion of effector tumor-specific CD $4^{+}$ $\mathrm{T}$ cells and to potentiate antitumor immune responses. Currently, a large number of clinical trials are using autophagy inhibition with chloroquine or hydroxychloroquine in combination with chemotherapy or other drugs for the treatment of multiple malignancies (61). However, the generalized effect of autophagy inhibition in the development of an antitumor immunity remains a caveat of such approaches. Targeted delivery of autophagy inhibitors (e.g., via nanoparticles) to MDSCs should be tested for the ability to generate immunity against tumors, and could pave the way for the design of novel therapeutic protocols in cancer.

\section{Methods}

Mice. C57BL/6 mice were purchased from the Jackson Laboratory, and $\operatorname{Atg} 5^{A L y s M}$ mice were generated by crossing Atg $5^{f / f l}$ mice (62) (RIKEN BioResource Center) and LysM ${ }^{\text {cre }}$ mice (Institute of Molecular Biology and Biotechnology Institute [IMBB]). OTII CD45.1 mice were provided by Federica Sallusto (Institute of Research in Biomedicine, Bellinzona, Switzerland). Female mice (8-10 weeks old) were used and maintained in the animal facility of the Biomedical Research Foundation of the Academy of Athens.

Cell lines. The melanoma cell line B16-F10 and the Lewis lung carcinoma cell line (LLC1) were provided by Aris Eliopoulos (Medical School, University of Crete, Heraklion, Greece). The B16-F10 cell line stably expressing ovalbumin with GFP (B16-F10-OVA.GFP) was provided by Caetano Reis e Sousa (The Francis Crick Institute, London, United Kingdom).

Solid tumor induction. Induction of solid tumors was performed as previously described (63). Briefly, C57BL/6, Atg $5^{4 L y s M}$, and Atg $5^{f / f l}$ mice were s.c. injected with $3 \times 10^{5}$ B16-F10 melanoma or LLC1 cells. Tumor volume was monitored from day 7 to day 15 and was calculated using the equation (length $\times$ width ${ }^{2}$ ) / 2. Analysis was performed 12-15 days after tumor inoculation.

Flow cytometry and cell sorting. For analysis of TILs, tumors were dissected and incubated for 45 minutes at $37^{\circ} \mathrm{C}$ in RPMI medium containing $0.1 \mathrm{mg} / \mathrm{ml}$ DNaseI (Sigma-Aldrich) and $0.2 \mathrm{mg} / \mathrm{ml} \mathrm{col-}$ lagenase D (Roche). Single-cell suspensions from TILs, peripheral blood mononuclear cells (PBMCs), spleen, or dLNs were stained with conjugated antibodies against mouse (catalog/clone): CD11c (117310/N418), Gr-1 (108408/RB6, 8C5), CD11b (101216/M1, 70), I-A ${ }^{b}$ (116406/AF6-120.1), Ly6G (127608/1A8), Ly6C (128032/HK1.4), CD4 (100406/GK1.5), CD8 (100722/53-6.7), CD45 (103132/30-F11), CD3 (100222/17A2), CD25 (101918/3C7), Va2 (127808/B20.1), NK1.1 (108710/PK136), CD115 (135511/AFS98), CD73 (127224/TY, 11.8), CTLA4 (106313/UC10-4B9), GITR (126312/DTA-1) CD40 (124625/3, 23) (Biolegend), pmTOR (12-9718-41/MRRBY), pAKT (17-9715-41/ S473), pS6 (12-9007-41/S235, S236) (eBioscience), and Lysosensor (L7535/DND189) (Molecular Probes). For Foxp3 intracellular staining, cells were fixed and stained using the Foxp3 Staining Set (antiFoxp3, catalog 12-4774-42/clone 150D; eBioscience) according to the manufacturer's instructions. For intracellular cytokine staining, CD $45^{+}$ cells sorted from tumors were incubated with $50 \mathrm{ng} / \mathrm{ml}$ PMA (SigmaAldrich), $2 \mu \mathrm{g} / \mathrm{ml}$ ionomycin (Sigma-Aldrich), and Golgi plug (1/1000; Becton Dickinson Biosciences) for 6 hours at $37^{\circ} \mathrm{C}$, and stained for IFN- $\gamma$ (catalog 505808/clone XMG1.2; Biolegend) using the BD Cytofix/Cytoperm Plus Fixation/Permeabilization kit (Becton Dickinson Biosciences). For intracellular phospho protein staining, cells were permeabilized with the intracellular Fixation \& Permeabilization buffer set (eBioscience) according to the manufacturer's instructions and stained with antibody against phosphor proteins. $\mathrm{CD} 11 \mathrm{c}^{-} \mathrm{CD} 11 \mathrm{~b}^{+} \mathrm{Gr}-1^{+}$ MDSCs, CD11c ${ }^{-}$CD $11 \mathrm{~b}^{+} \mathrm{Ly}_{6 \mathrm{C}^{+}}$M-MDSCs, CD ${ }^{+} \mathrm{CD} 25^{-} \mathrm{Va}^{+}$OTII T cells, CD11 $\mathrm{c}^{+} \mathrm{DCs}$, and $\mathrm{CD}^{+} \mathrm{T}$ cells were sorted on a FACS ARIA III (Becton Dickinson Biosciences). Cell purity was above 95\%. 
Immunofluorescence. Cells were seeded in coverslips pretreated with poly-L-lysine (Sigma-Aldrich) and fixed with $4 \%$ paraformaldehyde (Sigma-Aldrich) for 15 minutes in room temperature followed by 10 minutes of fixation with ice-cold methanol. Cells were permeabilized by using $0.1 \%$ saponin (Sigma-Aldrich), 2\% BSA, and stained with mouse anti-LC3 antibody (1:20; catalog 0231/clone 5F10; nanoTools), rat anti-LAMP-1 (1:400; sc-19992/1D4B; Santa Cruz Biotechnology), rabbit anti-p62 (1:500; PM045/SQSTM1; MBL), rabbit anti-phosphoULK1 (Ser556; 1:70; 5869S/D1H4; Cell Signaling Technology), mouse anti-LAMP-1 (1:400; sc-17768/E-5; Santa Cruz Biotechnology), rat anti-IA/IE (1:50; 14-5321-81/M5, 114.15.2; Biolegend), mouse antiRab7 (1:2000; ab50533/Rab7-117; Abcam), and goat anti-cathepsin D (1:100; sc-6486/C-20; Santa Cruz), then followed by incubation with Alexa Fluor 555 anti-mouse IgG (1:500; A28180; Invitrogen), Alexa Fluor 647 anti-rabbit IgG (1:200; A21245; Invitrogen), and Alexa Fluor 488 anti-rat IgG (1:250; A11006; Invitrogen). For visualization of the nuclei, DAPI (Sigma-Aldrich) was used. Samples were coverslipped with mowiol and visualized using a $\times 63$ oil lens in a Leica SP5 inverted confocal live cell imaging system.

The numbers of LC3 puncta/cell, p62 puncta/cell, Rab7 puncta/ cell, LAMP-1 puncta/cell, and cathepsin D puncta/cell were calculated using a macro developed in Fiji software as described (64). Colocalization of IA ${ }^{\mathrm{b}}$ with LAMP-1 and LC3 with p62 was calculated using crosscorrelation analysis with Volocity software (65).

For immunofluorescence staining of frozen tissues, tumors were embedded in OCT Tissue-Tek specimen matrix and cut in $7-\mu \mathrm{m}$-thick sections using Cryostar NX50 cryotome (Thermo Fisher Scientific) at $-20^{\circ} \mathrm{C}$. The sections were fixed in cold acetone for 10 minutes and left for at least 30 minutes at room temperature. Sections were blocked with $5 \%$ goat serum in TNT buffer $(20 \mathrm{mM}$ Tris, $\mathrm{pH} 7.6,0.9 \% \mathrm{NaCl}$, $0.05 \%$ Tween) for 30 minutes at room temperature and stained with rat anti-mouse CD206 (1:200; MR5D3; Santa Cruz) and rat antimouse CD4 (1:200; 14-0041-82/GK1.5; eBioscience) either at $37^{\circ} \mathrm{C}$ for 1 hour in a humidified chamber or at $4^{\circ} \mathrm{C}$ overnight, followed by staining with goat anti-rat IgG Alexa Fluor 555 (1:500; A-21434; Cell Signaling) for 30 minutes at room temperature. For visualization of the nuclei, DAPI ( $1 \mathrm{mg} / \mathrm{ml}, 1: 5,000$ in PBS) was used. Slides were mounted with fluorescent mounting medium (Dako) and visualized using a digital slide scanner (Zeiss Axio Scan).

All measurements were done only in the tumor area, not considering any positive signal in stroma-surrounding areas. The area positive for CD206 was measured with Fiji and is represented as percentage of tumor area positive for CD206. $\mathrm{CD}^{+}$cells were counted within the tumor area and represented as number of $\mathrm{CD} 4^{+}$cells per tumor.

Quantitative PCR analysis. Cells were lysed in Buffer RLT (Qiagen) and RNA was extracted using a Qiagen RNeasy mini kit according to the manufacturer's instructions. First-strand cDNA synthesis was performed using Superscript II reverse transcriptase (Invitrogen). qPCR was carried out using the iTaq Universal SYBR Green Supermix (BioRad). Relative expression of target genes was calculated by comparing them to the expression of the housekeeping gene Hprt. The following primers were used: mouse Atg5 forward, 5'-AGCTCTGGATGGGACTG-3'; Atg5 reverse 5'-CTCCGTCGTGGTCTGAT-3'; mouse Hprt forward, 5'-GTGAAACTGGAAAAGCCAAA-3'; Hprt reverse, 5'-GGACGCAGCAACTGACAT-3'; mouse Ciita forward, 5'-TGCGTGTGATGGATGTCCAG-3'; Ciita reverse, $5^{\prime}$-CCAAAGGGGATAGTGGGTGTC-3'; mouse $I A^{b}$ (Qiagen); mouse
March1 forward, 5'-AAGAGAGCCCACTCATCACACC-3'; March1 reverse, 5'-ATCTGGAGCTTTTCCCACTTCC-3'.

Suppression assays. Splenocytes from tumor-bearing mice were incubated with biotin anti-mouse Ly6C (1:200; HK1.4; Biolegend) followed by streptavidin microbeads (Miltenyi Biotec), and then Ly6C $\mathrm{C}^{+}$ cells were positively selected on a magnetic field according to the manufacturer's instructions (MACS separation columns MS, Miltenyi Biotec). Ly6 $\mathrm{C}^{+}$cells $\left(10^{6}\right)$ from $A \operatorname{tg} 5^{\Lambda L y s M}$ or Atg $5^{f / f l}$ mice were s.c. coinjected with $3 \times 10^{5} \mathrm{~B} 16-\mathrm{F} 10$ cells into C57BL/6 mice. The tumor volume was monitored from day 7 to day 15 .

For in vitro suppression assays, highly purified M-MDSCs were sorted from tumors of $\operatorname{Atg} 5^{4 L y s M}$ and $\operatorname{Atg} 5^{f / f l}$ mice and cultured in 96-well round-bottom plates with $1.5 \times 10^{5}$ whole CellTrace-labeled (10 $\mu \mathrm{m}$, Invitrogen) LN cells (LNCs) of naive C57BL/6 mice in a 1:2 ratio, in the presence of Dynabeads mouse T-activator CD3/CD8 (Life Technologies). Cells were analyzed 4 days later.

Antigen presentation assay. Magnetically isolated Ly6 $\mathrm{C}^{+}$cells $\left(10^{5}\right)$ were cultured in 1:1 ratio with CellTrace-labeled $\mathrm{CD} 4^{+} \mathrm{CD} 25^{-} \mathrm{Va} 2^{+} \mathrm{T}$ cells isolated from OTII naive mice in the presence of OVA $_{323-339}$ peptide $(20 \mu \mathrm{g} / \mathrm{ml}$, Caslo ApS). Cells were analyzed 4 days later.

Adoptive transfer experiments. Atg $5^{A L y s M}$ and Atg $5^{f / f l}$ mice received s.c. implantation of $3 \times 10^{5}$ B16-F10-OVA.GFP melanoma cells on the back. Seven days after injection, sorted $\mathrm{CD} 4^{+} \mathrm{CD} 25^{-} \mathrm{Va}^{+} \mathrm{T}$ cells $\left(10^{6}\right)$ from OTII naive mice labeled with CellTrace (50 $\mu \mathrm{m}$, Invitrogen) were i.v. transferred, and 4 days later, tdLNs were isolated and analyzed.

Preparation of tumor explant supernatants. Tumors from C57/BL6 mice were dissected at day 15 and single-cell suspensions were plated in 6-well plates $\left(10^{6}\right.$ cells $\left./ \mathrm{ml}\right)$. Supernatants were collected 16 hours later.

Lysosomal inhibition experiments. $\mathrm{Ly}^{6} \mathrm{C}^{+}$magnetically isolated cells $\left(2.5 \times 10^{5}\right)$ were plated in 96-well round-bottom plates and treated with tumor explant supernatants (TES, $20 \% \mathrm{vol} / \mathrm{vol}$ ) in the presence or absence of the inhibitors ammonium chloride $\left(\mathrm{NH}_{4} \mathrm{Cl}, 2 \mathrm{mM}\right.$, Sigma-Aldrich) and chloroquine diphosphate (CQ, $50 \mathrm{mM}$, SigmaAldrich) for 16 hours.

Measurement of lysosomal function (long-lived protein degradation assay). M-MDSCs were magnetically isolated from the spleens of B16-F10 melanoma cell-inoculated Atg $5^{4 L y s M}$ and Atg $5^{f / f l}$ mice. Lysosomal function was assessed with the long-lived protein degradation assay using $\left[{ }^{3} \mathrm{H}\right]$ leucine. In brief, $7 \times 10^{4}$ cells were plated in 48 -well plates. $\left[{ }^{3} \mathrm{H}\right]$ leucine (Perkin Elmer) was added to the culture media 24 hours later. The next day, the medium was replaced with starvationinducing medium and an excess of unlabeled leucine. After 6 hours, cells were treated with lysosomal inhibitors $\left(20 \mathrm{mM} \mathrm{NH}_{4} \mathrm{Cl}\right.$ and $20 \mu \mathrm{m}$ leupeptin or $100 \mathrm{nM}$ bafilomycin) (Sigma-Aldrich) or left untreated for 16 hours. For precipitation of the degraded proteins, aliquots of culture supernatants were treated with $20 \%$ trichloroacetic acid and BSA (20 $\mathrm{mg} / \mathrm{ml}$ ). For isolation of nondegraded proteins (proteins in media and cell lysates), cells were lysed with a mild lysis buffer containing 0.1 $\mathrm{N} \mathrm{NaOH}$ and $0.1 \% \mathrm{wt} / \mathrm{vol}$ sodium deoxycholate. Counts per minute (cpm) were obtained using a beta counter. The protein degradation was calculated as degraded proteins / (nondegraded proteins + lysed cells).

RNA sequencing analysis. M-MDSCs were isolated from the spleens of B16-F10 melanoma cell-inoculated $\operatorname{Atg} 5^{4 L y s M}$ and Atg $5^{f / / l}$ mice using magnetic beads. RNA was extracted with the Macherey-Nagel Nucleo-spin RNA kit. RNA sequencing was employed and singleend 75-bp-length reads were generated. Data were aligned to the mouse genome ( $\mathrm{mm} 9$ version) with the TopHat2 algorithm. HT-seq 
and DESeq algorithm were used in order to measure gene expression and identify differential expression between the 2 groups of patients. Genes with a $P$ value $\leq 0.05$ and fold change $\geq 1.5$ or $\leq-1.5$ were considered to be up- and downregulated, respectively. Gene ontology analysis, pathway annotation, transcription factor enrichment, and comparison with various immunological and oncogenic gene signatures were performed with the use of DAVID knowledge base, Ingenuity Pathway Analysis software, and Molecular Signature Database (MSigDB) from Broad Institute (SRA accession number PRJNA395259).

Silencing of March1 by siRNA. Splenocytes from tumor-bearing C57BL/6 mice were incubated with biotin anti-mouse Ly6C (1:200; HK1.4; Biolegend) followed by streptavidin microbeads (Miltenyi Biotec), and then $\mathrm{Ly}_{6} \mathrm{C}^{+}$cells were positively selected on a magnetic field according to the manufacturer's instructions (MACS separation columns MS, Miltenyi Biotec). Ly6 $\mathrm{C}^{+}$cells were seeded in 12-well plates $\left(1.5 \times 10^{6}\right)$, cultured with $250 \mathrm{ng} / \mathrm{ml} \mathrm{LPS}$, and transfected with $20 \mu \mathrm{l}$ $(10 \mu \mathrm{M})$ of either March1 siRNA cocktail (Santa Cruz Biotechnology, sc-106199) or scramble si-RNA sequence (Santa Cruz Biotechnology, sc-37007) using Lipofectamine 2000 (Thermo Fisher Scientific, 11668019) according to the manufacturer's protocol. Forty-eight hours after transfection, cells were washed, harvested, and tested with qPCR for knockdown efficiency. The expression of MHC II in transfected cells was assessed with FACS. Transfected Ly6C $\mathrm{C}^{+}$cells $\left(4 \times 10^{5}\right)$, with either scramble or si-March1, were s.c. coinjected with $3 \times 10^{5}$ B16-F10 cells into C57BL/6 mice. The tumor volume was monitored from day 7 to day 12 .

Human subjects and isolation of MDSCs from peripheral blood. Peripheral blood from patients with melanoma (stage IV) and healthy individuals was collected in EDTA-coated tubes prior to systemic treatment. PBMCs were isolated using Histopaque 1077 (Sigma-Aldrich) (400 $\mathrm{g}, 30$ minutes, room temperature), and washed and stained with antibodies against human CD14 (325604/HCD14), CD33 (303404/ WM53), CD15 (323018/W6D3), and HLA-DR (307616/L243) (Biolegend) prior to MDSC sorting. Sorted MDSCs were processed for autophagy pathway analysis with confocal microscopy.

Statistics. Statistical analyses were performed using an unpaired 2-tailed Student's $t$ test. Two-way ANOVA statistical tests were applied in experiments with multiple comparisons. Data are mean \pm SEM. Differences were considered statistically significant at $P<0.05$. All data were analyzed using GraphPad Prism v5 software.
Study approval. Patients with melanoma (stage IV) and healthy individuals were recruited through the Oncology Department, University Hospital "Laiko" (Athens, Greece). The Clinical Research Ethics Board of National and Kapodistrian University of Athens, Medical School, University Hospital "Laiko" (Athens, Greece) approved this study. Informed consent was obtained from all patients and healthy individuals prior to sample collection. All procedures in mice were in accordance with institutional guidelines and were reviewed and approved by the Greek Federal Veterinary Office (Athens, Greece, protocol 1474).

\section{Author contributions}

$\mathrm{TA}$ and $\mathrm{AH}$ designed and performed experiments, analyzed data, generated figures, and wrote the manuscript. $\mathrm{KM}, \mathrm{RMB}$, and $\mathrm{AB}$ performed experiments, analyzed data, and generated figures. SS and BW performed immunohistochemical analysis and participated in interpretation of data. AP and MX assisted with experiments and analyzed data. HG performed clinical evaluation of the patients and provided human specimens. PV designed and supervised the study, performed data analysis, and wrote the manuscript.

\section{Acknowledgments}

We thank Katerina Girtzimanaki and George Panagopoulos for assisting with experiments and for providing technical advice, Anastasia Apostolidou and Arianna Gavriil for cell sorting, and Stamatis Pagakis and Eleni Rigana for providing assistance with confocal microscopy and quantification of confocal images. This work was supported by grants from the Greek General Secretariat of Research and Technology (Aristeia II 3468 to P.V.), COST Action Mye-EUNITER (BM1404, http://www.mye-euniter.eu), and Trans Autophagy (CA15138, http://cost-transautophagy.eu), as part of the European Union Framework Program Horizon 2020. TA and AH are supported by IKY Fellowships of Excellence for Postgraduate Studies in Greece, Siemens Programme.

Address correspondence to: Panayotis Verginis, Center of Clinical, Experimental Surgery \& Translational Research, Biomedical Research Foundation, Academy of Athens, 4 Soranou Efessiou Street, 11527 Athens, Greece. Phone: 30.210.6597516; Email: pverginis@bioacademy.gr.
1. Gentles AJ, et al. The prognostic landscape of genes and infiltrating immune cells across human cancers. Nat Med. 2015;21(8):938-945.

2. Chen DS, Mellman I. Oncology meets immunology: the cancer-immunity cycle. Immunity. 2013;39(1):1-10.

3. Ioannou $\mathrm{M}$, et al. Crucial role of granulocytic myeloid-derived suppressor cells in the regulation of central nervous system autoimmune disease. JImmunol. 2012;188(3):1136-1146.

4. Gabrilovich DI, Nagaraj S. Myeloid-derived suppressor cells as regulators of the immune system. Nat Rev Immunol. 2009;9(3):162-174.

5. Talmadge JE, Gabrilovich DI. History of myeloidderived suppressor cells. Nat Rev Cancer. 2013;13(10):739-752.

6. Hatziioannou A, Alissafi T, Verginis P. Myeloidderived suppressor cells and $\mathrm{T}$ regulatory cells in tumors: unraveling the dark side of the force. JLeukoc Biol. 2017;102(2):407-421.

7. Marvel D, Gabrilovich DI. Myeloid-derived suppressor cells in the tumor microenvironment: expect the unexpected. J Clin Invest. 2015;125(9):3356-3364.

8. Mizushima N, et al. Mouse Apg16L, a novel WDrepeat protein, targets to the autophagic isolation membrane with the Apg12-Apg5 conjugate. J Cell Sci. 2003;116(Pt 9):1679-1688.

9. Wilson WR, Hay MP. Targeting hypoxia in cancer therapy. Nat Rev Cancer. 2011;11(6):393-410.

10. White E. The role for autophagy in cancer. JClin Invest. $2015 ; 125(1): 42-46$.

11. Bellot G, et al. Hypoxia-induced autophagy is mediated through hypoxia-inducible factor induction of BNIP3 and BNIP3L via their BH3 domains. Mol Cell Biol. 2009;29(10):2570-2581.
12. Corzo CA, et al. HIF- $1 \alpha$ regulates function and differentiation of myeloid-derived suppressor cells in the tumor microenvironment. J Exp Med. 2010;207(11):2439-2453.

13. Rouschop KM, et al. The unfolded protein response protects human tumor cells during hypoxia through regulation of the autophagy genes MAP1LC3B and ATG5.J Clin Invest. 2010;120(1):127-141.

14. Parker KH, Horn LA, Ostrand-Rosenberg S. High-mobility group box protein 1 promotes the survival of myeloid-derived suppressor cells by inducing autophagy. J Leukoc Biol. 2016;100(3):463-470.

15. Ogata M, et al. Autophagy is activated for cell survival after endoplasmic reticulum stress. Mol Cell Biol. 2006;26(24):9220-9231.

16. Yorimitsu T, Nair U, Yang Z, Klionsky DJ. Endo- 
plasmic reticulum stress triggers autophagy. J Biol Chem. 2006;281(40):30299-30304.

17. Condamine T, et al. ER stress regulates myeloid-derived suppressor cell fate through TRAIL-R-mediated apoptosis. JClin Invest. 2014;124(6):2626-2639.

18. Thevenot PT, et al. The stress-response sensor chop regulates the function and accumulation of myeloid-derived suppressor cells in tumors. Immunity. 2014;41(3):389-401.

19. Scherz-Shouval R, Elazar Z. Regulation of autophagy by ROS: physiology and pathology. Trends Biochem Sci. 2011;36(1):30-38.

20. Klionsky DJ, et al. Guidelines for the use and interpretation of assays for monitoring autophagy (3rd edition). Autophagy. 2016;12(1):1-222.

21. Kabeya Y, et al. LC3, a mammalian homologue of yeast Apg8p, is localized in autophagosome membranes after processing. EMBO J. 2000;19(21):5720-5728.

22. Pankiv S, et al. p62/SQSTM1 binds directly to Atg8/LC3 to facilitate degradation of ubiquitinated protein aggregates by autophagy. J Biol Chem. 2007;282(33):24131-24145.

23. Martina JA, Chen Y, Gucek M, Puertollano R. MTORC1 functions as a transcriptional regulator of autophagy by preventing nuclear transport of TFEB. Autophagy. 2012;8(6):903-914.

24. Ganley IG, Lam du H, Wang J, Ding X, Chen S, Jiang X. ULK1.ATG13.FIP200 complex mediates mTOR signaling and is essential for autophagy. J Biol Chem. 2009;284(18):12297-12305.

25. Reggiori F, Komatsu M, Finley K, Simonsen A. Selective types of autophagy. Int JCell Biol. 2012;2012:156272.

26. Quatromoni JG, Eruslanov E. Tumor-associated macrophages: function, phenotype, and link to prognosis in human lung cancer. Am J Transl Res. 2012;4(4):376-389.

27. Pan PY, et al. Immune stimulatory receptor CD40 is required for $\mathrm{T}$-cell suppression and $\mathrm{T}$ regulatory cell activation mediated by myeloidderived suppressor cells in cancer. Cancer Res. 2010;70(1):99-108.

28. Teichgräber V, et al. Ceramide accumulation mediates inflammation, cell death and infection susceptibility in cystic fibrosis. Nat Med. 2008;14(4):382-391.

29. Wang T, Ming Z, Xiaochun W, Hong W. Rab7: role of its protein interaction cascades in endolysosomal traffic. Cell Signal. 2011;23(3):516-521.

30. Cho KJ, Walseng E, Ishido S, Roche PA. Ubiquitination by March-I prevents MHC class II recycling and promotes $\mathrm{MHC}$ class II turnover in antigen-presenting cells. Proc Natl Acad Sci U S A. 2015;112(33):10449-10454.

31. Melero I, Rouzaut A, Motz GT, Coukos G. T-cell and NK-cell infiltration into solid tumors: a key limiting factor for efficacious cancer immunotherapy. Cancer Discov. 2014;4(5):522-526.

32. Marzo AL, et al. Tumor-specific CD4+ T cells have a major "post-licensing" role in CTL mediated anti-tumor immunity. JImmunol.
2000;165(11):6047-6055

33. Bos R, Sherman LA. CD4+ T-cell help in the tumor milieu is required for recruitment and cytolytic function of CD8+ T lymphocytes. Cancer Res. 2010;70(21):8368-8377.

34. Muranski P, et al. Tumor-specific Th17-polarized cells eradicate large established melanoma. Blood. 2008;112(2):362-373.

35. Martin-Orozco N, et al. T helper 17 cells promote cytotoxic $\mathrm{T}$ cell activation in tumor immunity. Immunity. 2009;31(5):787-798.

36. Quezada SA, et al. Tumor-reactive CD4(+) T cells develop cytotoxic activity and eradicate large established melanoma after transfer into lymphopenic hosts. J Exp Med. 2010;207(3):637-650.

37. Nagaraj S, Nelson A, Youn JI, Cheng P, Quiceno D, Gabrilovich DI. Antigen-specific CD4(+) T cells regulate function of myeloid-derived suppressor cells in cancer via retrograde MHC class II signaling. Cancer Res. 2012;72(4):928-938.

38. Poschke I, Mougiakakos D, Hansson J, Masucci GV, Kiessling R. Immature immunosuppressive CD14+HLA-DR-/low cells in melanoma patients are Stat3hi and overexpress CD80, CD83, and DC-sign. Cancer Res. 2010;70(11):4335-4345.

39. Filipazzi $P$, et al. Identification of a new subset of myeloid suppressor cells in peripheral blood of melanoma patients with modulation by a granulocyte-macrophage colony-stimulation factor-based antitumor vaccine. JClin Oncol. 2007;25(18):2546-2553.

40. Zea AH, et al. Arginase-producing myeloid suppressor cells in renal cell carcinoma patients: a mechanism of tumor evasion. Cancer Res. 2005;65(8):3044-3048.

41. Serafini P, Mgebroff S, Noonan K, Borrello I. Myeloid-derived suppressor cells promote crosstolerance in B-cell lymphoma by expanding regulatory T cells. Cancer Res. 2008;68(13):5439-5449.

42. Hoechst B, et al. A new population of myeloidderived suppressor cells in hepatocellular carcinoma patients induces CD4(+)CD25(+)Foxp3(+) T cells. Gastroenterology. 2008;135(1):234-243.

43. Delamarre L, Pack M, Chang H, Mellman I, Trombetta ES. Differential lysosomal proteolysis in antigen-presenting cells determines antigen fate. Science. 2005;307(5715):1630-1634.

44. Tze LE, et al. CD83 increases MHC II and CD86 on dendritic cells by opposing IL-10-driven MARCH1-mediated ubiquitination and degradation. J Exp Med. 2011;208(1):149-165.

45. Roche PA, Furuta K. The ins and outs of MHC class II-mediated antigen processing and presentation. Nat Rev Immunol. 2015;15(4):203-216.

46. van Niel G, et al. Dendritic cells regulate exposure of MHC class II at their plasma membrane by oligoubiquitination. Immunity. 2006;25(6):885-894.

47. Matsuki Y, et al. Novel regulation of $\mathrm{MHC}$ class II function in B cells. EMBO J. 2007;26(3):846-854.

48. Zhu J, Huang X, Yang Y. Myeloid-derived suppressor cells regulate natural killer cell response to adenovirus-mediated gene transfer. J Virol.
2012;86(24):13689-13696.

49. Büchau AS, et al. The host defense peptide cathelicidin is required for NK cell-mediated suppression of tumor growth. JImmunol. 2010;184(1):369-378

50. Mauti LA, et al. Myeloid-derived suppressor cells are implicated in regulating permissiveness for tumor metastasis during mouse gestation. J Clin Invest. 2011;121(7):2794-2807.

51. Korolchuk VI, et al. Lysosomal positioning coordinates cellular nutrient responses. Nat Cell Biol. 2011;13(4):453-460.

52. Yan C, Ding X, Dasgupta N, Wu L, Du H. Gene profile of myeloid-derived suppressive cells from the bone marrow of lysosomal acid lipase knockout mice. PLoS ONE. 2012;7(2):e30701.

53. Ding X, Zhang W, Zhao T, Yan C, Du H. Rab7 GTPase controls lipid metabolic signaling in myeloid-derived suppressor cells. Oncotarget. 2017;8(18):30123-30137.

54. Ko JS, et al. Direct and differential suppression of myeloid-derived suppressor cell subsets by sunitinib is compartmentally constrained. Cancer Res. 2010;70(9):3526-3536.

55. Condamine T, Gabrilovich DI. Molecular mechanisms regulating myeloid-derived suppressor cell differentiation and function. Trends Immunol. 2011;32(1):19-25.

56. Schaaf MB, et al. The autophagy associated gene, ULK1, promotes tolerance to chronic and acute hypoxia. Radiother Oncol. 2013;108(3):529-534.

57. Mazure NM, Pouysségur J. Hypoxia-induced autophagy: cell death or cell survival? Curr Opin Cell Biol. 2010;22(2):177-180.

58. Liu G, et al. SIRT1 limits the function and fate of myeloid-derived suppressor cells in tumors by orchestrating HIF-1 $\alpha$-dependent glycolysis. Cancer Res. 2014;74(3):727-737.

59. Kumar V, et al. CD45 phosphatase inhibits STAT3 transcription factor activity in myeloid cells and promotes tumor-associated macrophage differentiation. Immunity. 2016;44(2):303-315.

60. Kimmelman AC. The dynamic nature of autophagy in cancer. Genes Dev. 2011;25(19):1999-2010.

61. Towers CG, Thorburn A. Therapeutic Targeting of autophagy. EBioMedicine. 2016;14:15-23.

62. Hara T, et al. Suppression of basal autophagy in neural cells causes neurodegenerative disease in mice. Nature. 2006;441(7095):885-889.

63. Hatzioannou A, Nayar S, Gaitanis A, Barone F, Anagnostopoulos C, Verginis P. Intratumoral accumulation of podoplanin-expressing lymph node stromal cells promote tumor growth through elimination of CD4+ tumorinfiltrating lymphocytes. Oncoimmunology. 2016;5(9):e1216289.

64. Alissafi T, et al. Tregs restrain dendritic cell autophagy to ameliorate autoimmunity. J Clin Invest. 2017;127(7):2789-2804.

65. Costes SV, Daelemans D, Cho EH, Dobbin Z, Pavlakis G, Lockett S. Automatic and quantitative measurement of protein-protein colocalization in live cells. Biophys J. 2004;86(6):3993-4003. 